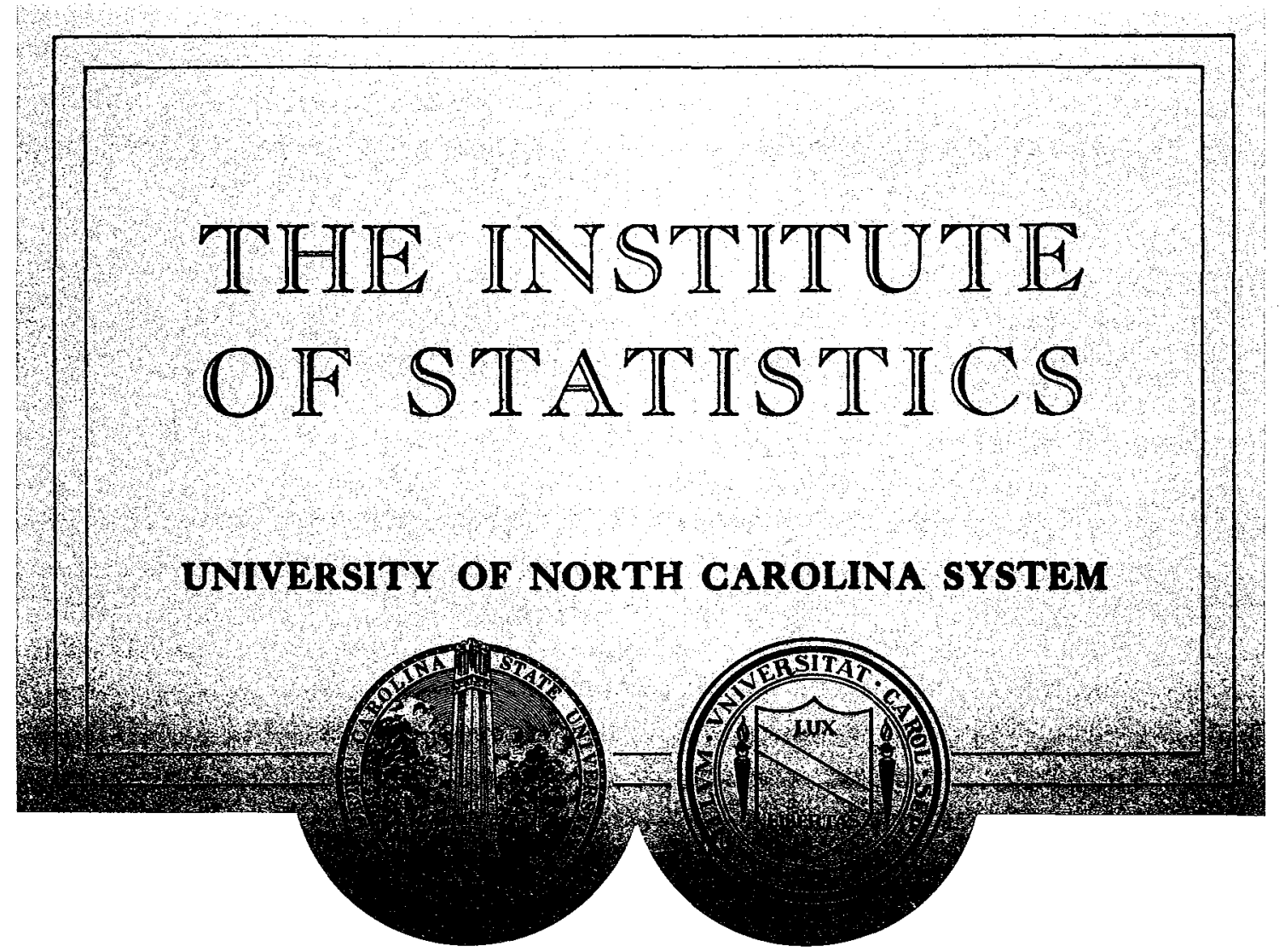

ASSAYS FOR RECOMBINANT PROTEINS: A PROBLEM IN NONLINEAR CALIBRATION

by

David M. Giltinan and Marie Davidian

Institution of Statistics Mimeograph Series No. 2223

May, 1992 


MIMEO David M. Giltian and
SERIES Marie Davidian
\#2223 ASSAYS FOR RECOMBINANT
PROTEINS: A PROBLEM IN
NONIINER CALIBRATION

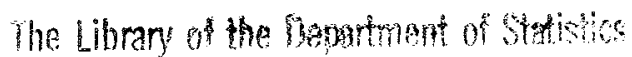
North Carolina State Unwersity 


\section{Assays for Recombinant Proteins: A Problem in Nonlinear Calibration ${ }^{1}$}

\author{
David M. Giltinan \\ Genentech, Inc.
}

\author{
Marie Davidian \\ North Carolina State University
}

May 1992

\footnotetext{
${ }^{1}$ Submitted to Case Studies in Biometry. Corresponding author: David M. Giltinan, Biostatistics Department, Genentech, Inc., 460 Point San Bruno Blvd., South San Francisco CA 94080 USA, 415-225-2296, giltinan@gene.com (internet).
} 


\section{Introduction}

A recurring challenge in the development of recombinant proteins for therapeutic use is the derivation of suitable assays for measuring protein concentrations in serum or plasma. Techniques such as HPLC, which are appropriate for low molecular weight drugs, are typically unsuitable for assaying macromolecules such as proteins, particularly in complex biological matrices. Instead, methods used to quantify protein concentration frequently exploit the specificity of immunoassay techniques such as radioimmunoassay (RIA), immunoradiometric assay (IRMA), or enzyme-linked immunosorbent assay (ELISA). Such methods can often achieve sensitivity in the $\mathrm{pg} / \mathrm{ml}$ range. Clark and Engvall (1980, chap. 8) contains an excellent overview of immunologically based assay techniques, that is, methods based on antigen-antibody reactions.

The data shown in Table 1 were generated during development of an ELISA assay for the recombinant enzyme human DNase in rat serum. For each of eleven experiments, duplicate absorbance (optical density) measurements were obtained at each of several known concentrations of standard. Figure 1 shows profiles for each run, where responses at zero concentration are plotted at two dilutions below the lowest non- zero dose, by convention. The four-parameter logistic sigmoid

$$
f(x, \beta)=\beta_{1}+\left(\beta_{2}-\beta_{1}\right) /\left[1+\exp \left\{\beta_{4}\left(\log x-\beta_{3}\right)\right\}\right]
$$

(Rodbard and Frazier, 1975) provides an appropriate characterization of dose-response, where the parameters may vary across experiments. In any particular assay run, the primary focus is on calibration of DNase concentrations in unknown samples, based on an appropriate fitted standard curve.

While immunoassays are extremely useful in quantifying protein concentrations, a requirement is the development of an assay which provides a measure of the biological activity of the protein, such as an in-vitro cell-based bioassay. Such an assay is required for regulatory purposes, and reflects the fact that the degree of antigen binding exhibited by a polypeptide such as an antibody does not necessarily provide a good indication of its biological activity.

Table 2 shows results for an assay of this type. The values represent dose-response data obtained for standard concentrations in nine runs of a bioassay for the recombinant hormone human relaxin. The assay is based on increased generation and release of intracellular cAMP by normal human uterine endometrial (NHE) cells in the presence of relaxin (Fei et al., 
1990). For each of the nine assay runs presented here, triplicate measurements of cAMP concentrations were determined by radioimmunoassay following incubation of a fixed number of NHE cells with one of seven known concentrations of relaxin. A single zero-standard response value was also available for each plate. Figure 2 illustrates the concentrationresponse data for the standard for these runs, where again, by convention, response at zero concentration is plotted at two dilutions below the lowest standard. The plots indicate that the four-parameter logistic model provides a reasonable representation of the dose-response relationship, where regression parameters may vary from run to run.

For both the DNase ELISA and the relaxin bioassay, calibration of protein concentrations in unknown samples, based on a fitted standard curve, is the primary objective. In the next section, we describe a general parametric framework to accommodate calibration inference. Within this framework, we discuss three specific practical issues arising in nonlinear calibration:

1. Characterization of the intra-assay variability in calibrated concentrations.

2. Construction of confidence intervals about calibrated concentrations.

3. Use of empirical Bayes methods in calibration.

The methods and issues discussed will be illustrated by reference to the data sets described above, and by limited simulation work.

\section{A Nonlinear Mixed Effects Model}

In developing a general model for inference, we suppose that data pairs $\left(x_{i j}, y_{i j}\right)$ are available from $n$ runs of the assay, where $y_{i j}$ denotes the observed response at one of the concentrations $x_{i j}$ for the $i$ th assay run, $j=1, \ldots, m_{i}$. A natural parametric framework for inference is provided by the nonlinear mixed effects model

$$
y_{i j}=f\left(x_{i j}, \beta_{i}\right)+\sigma g\left\{f\left(x_{i j}, \beta_{i}\right), \theta\right\} \epsilon_{i j} .
$$

In this equation, the subscript $i$ indexes run number within a series of assay runs, and $j$ indexes measurements within a run. Replicate measurements are usually taken at some or all of the concentrations for a given run; this is not explicitly highlighted in Equation 2. 
Response, $y$, is related to the known standard concentration, $x$, according to the nonlinear regression function $f$. Choice of the four parameter logistic function, given by Equation 1, is fairly standard for $f$ in the context of immunoassay or bioassay (Rodbard and Frazier, 1975; Finney, 1976). Conceptually, however, any appropriate function $f$ of a general $p$-dimensional parameter vector may be substituted in Equation 2.

In the particular case of the logistic function, the four parameters have the following interpretation:

$\beta_{1}:$ response at zero dose

$\beta_{2}:$ response at infinite dose

$\beta_{3}: \log$ EC50 value; $\log$ of the dose which gives a response midway between $\beta_{1}$ and $\beta_{2}$, location of the inflection point

$\beta_{4}$ : slope parameter, measuring steepness of the concentration-response curve.

It is clear from the relaxin data (Figure 2) that regression parameter values can change considerably from run to run. This interassay variability is accommodated in the model by considering $\beta_{i}$ to be random vectors from a distribution with mean $\beta$ and covariance matrix $\Sigma$; the usual specification is that of a $p$-variate normal distribution. The intra-assay errors $\epsilon_{i j}$ are assumed to be independently and identically distributed with zero mean and variance one, independently of the $\beta_{i}$. The further assumption of normality of the $\epsilon_{i j}$ is often reasonable.

A routine phenomenon in assay data is heteroscedasticity in the intra-assay response. Typically, this is manifested as a systematic relationship between the intra-assay variability and the response level. This is accounted for in Equation 2 by the scale parameter $\sigma$ and a variance function $g$ depending on the conditional mean $f\left(x_{i j}, \beta_{i}\right)$ and a $q$-dimensional parameter vector $\theta$, that is:

$$
E\left(y_{i j} \mid \beta_{i}\right)=f\left(x_{i j}, \beta_{i}\right) ; \quad \operatorname{Var}\left(y_{i j} \mid \beta_{i}\right)=\sigma^{2} g^{2}\left\{f\left(x_{i j}, \beta_{i}\right), \theta\right\} .
$$

The variance function $g$ is chosen to reflect the likely character of intra-assay response variability. In Equation $3, g, \sigma$, and $\theta$ are assumed common across assay runs. This reflects a belief that the pattern of variablity is consistent across assays, which seems reasonable provided assay procedures have stabilized. The appropriateness of this assumption should 
be weighed in any given situation; it is certainly conceptually possible to allow $g, \sigma$, and $\theta$ to vary with $i$, though this will complicate inference on variance parameters; in particular, the methods described in Section 3 below will not apply.

In the case of assay data, variance is often modeled as a function of the mean $\mu_{i j}=$ $f\left(x_{i j}, \beta_{i}\right)$; a common choice is the power of the mean variance model

$$
g(\mu, \theta)=\mu^{\theta}
$$

(Rodbard and Frazier, 1975; Finney, 1976). Other possibilities include the exponential model; $g(\mu, \theta)=\exp (\mu \theta)$, the components of variance model $g(\mu, \theta)=\theta_{1}+\mu^{\theta_{2}}$. A general discussion may be found in Carroll and Ruppert (1988, chap. 3). This framework allows considerable flexibility in characterizing intra-assay variability through specification of $g$ and $\theta$. The form of $g$ is assumed to be known, but the analyst may be unable to specify an appropriate value for $\theta$. For example, in developing a new assay, the variability may not be well understood; the power of the mean model may be reasonable, but common choices, such as $\theta=0.5$ or 1.0 may be inappropriate (Finney, 1976). In more complicated models, it may be difficult to specify a value for $\theta$ a priori. In such cases, a reasonable strategy is to estimate $\theta$ from the data; we discuss methods for doing this in Section 3 below.

Why pay so much attention to the intra-assay variability in the response? It does affect appropriate choice of weighting scheme in fitting the standard curve, and thus affects the regression parameter estimate, but suboptimal weighting due to some misspecification of either the form of $g$ or the value of $\theta$ is unlikely to have a serious impact on the estimate of $\beta_{i}$ for a given run. A more compelling reason is that it directly affects one's ability to assess the precision of calibration correctly, as the following development shows.

A standard technique for evaluating the intra-assay precision associated with calibration of an unknown sample in a given experiment is to construct a precision profile (Ekins and Edwards, 1983). Such a profile is simply a plot of the estimated precision of an estimated concentration against concentration across the assay range. Several methods of constructing a precision profile are possible, depending on the measure of precision used (standard error or coefficient of variation) and the method used to calculate it. For concreteness, we focus on one computational scheme here, but the issues raised will also arise in the case of other calculation methods.

Let $h(y, \beta)$ denote the inverse of the regression function $f$, specified in Equation 2. In 
the particular case of the four-parameter logistic function, $h$ has the form

$$
x=h(y, \beta)=\exp \left[\beta_{3}+\log \left\{\left(\beta_{2}-y\right) /\left(y-\beta_{1}\right)\right\} / \beta_{4}\right]
$$

Let $h_{y}(y, \beta)$ and the $p$-variate vector-valued function $h_{\beta}(y, \beta)$ represent the derivatives of $h$ with respect to $y$ and $\beta$, respectively. Then if $y_{0}$ represents the average response for $r$ replicates of an unknown sample at concentration $x_{0}$, the estimated value of $x_{0}$ is given by $\tilde{x}_{0}=h\left(y_{0}, \tilde{\beta}_{i}\right)$, where $\tilde{\beta}_{i}$ is an estimate of $\beta_{i}$ with estimated covariance matrix $V_{i}$. An estimated approximate variance for $\tilde{x}_{0}$ may be obtained by a standard Taylor series linearization and is given by

$$
\operatorname{Var}\left(\tilde{x}_{0}\right)=h_{y}^{2}\left(y_{0}, \tilde{\beta}_{i}\right) \tilde{\sigma}^{2} g^{2}\left(y_{0}, \tilde{\theta}\right) / r+h_{\beta}^{\prime}\left(y_{0}, \tilde{\beta}_{i}\right) V_{i} h_{\beta}\left(y_{0}, \tilde{\beta}_{i}\right)
$$

where $(\tilde{\sigma}, \tilde{\theta})$ are estimates of the intra-assay variance parameters. The first term in Equation 6 reflects uncertainty in the measurement of $y_{0}$ and may be expected to dominate the second term, which corresponds to uncertainty in the fitted standard curve. For some assays, however, the contribution of the second term may be substantial; in such cases, the widespread practice in the assay and clinical chemistry literature of taking only the first term of Equation 6 may result in highly optimistic estimates of intra-assay precision.

To illustrate the importance of a good understanding of the correct value of the variance parameter $\theta$, consider the profiles shown in Figure 3. These represent precision profiles of $\left\{\operatorname{Var}\left(\tilde{x}_{0}\right)\right\}^{1 / 2} / \tilde{x}_{0}$ versus $\tilde{x}_{0}$, based on run \#1 of the relaxin bioassay, with the variance being calculated according to Equation 6 using the power of the mean variance model (Equation 4) under the assumptions $\theta=0,0.5$ and 1.0 , respectively, and triplicate response measurements for the unknown sample in all cases $(r=3)$. The assessment of intra-assay precision diverges widely, according to our belief about the appropriate value of $\theta$. Not all three can be correct; our point here is simply that accurate knowledge of $\theta$ is necessary in order to construct an accurate precision profile. In the next section, we discuss methods of estimating the appropriate value of $\theta$, based on data from a number of assay runs.

We have presented the model specified by Equations 2 and 3 as a basis for nonlinear calibration inference based on data from several assay runs. It is worth noting that this model framework is also useful in describing other kinds of data. In particular, a heteroscedastic nonlinear mixed effects model is useful in the context of population pharmacokinetic analysis, where the same three model features occur: (1) a nonlinear regression function, (2) regression 
parameters which vary from subject to subject, and (3) heterogeneity of intrasubject variation, often exhibiting a systematic dependence upon mean response. The pharmacokinetics literature makes extensive reference to this model framework; an overview may be found in Beal and Sheiner (1982). Another area where nonlinear mixed effects modeling can prove helpful is in growth curve analysis; see Davidian and Giltinan (1992a) for an example.

\section{Characterizing Intra-assay Variability}

In this section we discuss methods for estimating the variance parameters $(\sigma, \theta)$, given the model specifications described by Equations 2 and 3. The numerical illustrations below involve the specific choice of $f$ as the four-parameter logistic function and $g$ as the power of the mean variance function. The methods apply for general choices of $f$ and $g$, however, and throughout the exposition we continue to regard $f$ and $g$ as general functions of $x$, the regression parameter $\beta_{i}$, and, in the case of $g, 0$. The literature on variance function estimation is extensive; Carroll and Ruppert (1988, chap. 3) provide a comprehensive review. Our discussion here focuses mainly on techniques described in Davidian and Giltinan (1992a,b).

Several techniques for variance function estimation have been proposed for the case where data are available from only a single assay run. This represents a convenient point of departure for expository purposes, and we begin by discussing this situation. We emphasize at the outset, however, that in almost all cases, data from a single assay run will be insufficient to provide a reliable characterization of intra-assay variability, because estimation of second moment properties is inherently more difficult than estimation of means. However, certain estimation methods for the case of data from a single assay extend naturally to deal with the case of several assays, and it is this extension, described below, which proves most useful in practice.

Suppose, for the moment, that data are available from only one assay run. Several methods have been proposed for estimating $\theta$ and $\beta_{i}$ based on data from run $i$ only. One possibility is simultaneous estimation of $\left(\beta_{i}, \sigma, \theta\right)$ by normal theory maximum likelihood. This method is sometimes referred to as extended least squares (ELS) in the pharmacokinetic literature (Peck, et al., 1984). While this approach is theoretically optimal under the assumption of normality, the efficiency advantage is highly dependent on the normality assumption being exactly correct (Carroll and Ruppert, 1988, chap. 2). In addition, Carroll and Ruppert (1982) and van Houwelingen (1988) point out that slight misspecification of the form of the 
variance function can lead not only to poor variance estimation, but also to deterioration of the estimate of $\beta_{i}$. Furthermore, this approach does not extend naturally to handling data from several assays; the computational burden of simultaneous maximization of a suitable objective function in $\left(\beta_{i}, \sigma, \theta\right)$ seems prohibitive for more than a moderate number of assay runs.

A very general alternative approach is provided by the class of methods for estimation of $\left(\beta_{i}, \sigma, \theta\right)$ known as generalized least squares (GLS). These methods can be characterized by the following scheme:

1. Estimate $\beta_{i}$ by a preliminary unweighted fit, for example, ordinary least squares (OLS).

2. Use residuals from the preliminary fit to estimate $\theta$ and $\sigma$ and form estimated weights based on the estimates of $\theta$ and $\beta_{i}$.

3. Use the weights from step 2 to re-estimate $\beta_{i}$ by weighted regression. Treating the resulting estimate as a new preliminary estimate, return to step 2 .

Step 2 is nonspecific and can be implemented in practice in several ways. We focus on three methods, all based on transformations of absolute residuals from a preliminary fit, and all relatively easy to compute.

Given a preliminary estimate $\beta_{i}^{p}$ of $\beta_{i}$, the method of pseudolikelihood (PL) minimizes in $\sigma$ and $\theta$

$$
P L_{i}\left(\beta_{i}^{p}, \sigma, \theta\right)=\sum_{j=1}^{m_{i}}\left(\left\{y_{i j}-f\left(x_{i j}, \beta_{i}^{p}\right)\right\}^{2} /\left[\sigma^{2} g^{2}\left\{f\left(x_{i j}, \beta_{i}^{p}\right), \theta\right\}\right]+\log \left[\sigma^{2} g^{2}\left\{f\left(x_{i j}, \beta_{i}^{p}\right), \theta\right\}\right]\right) .
$$

This corresponds to maximizing the normal loglikelihood, evaluated at $\beta_{i}^{p}$, in the parameters $\sigma$ and $\theta$. Note that this procedure does not give the same results as joint maximization of the likelihood in $\beta_{i}, \sigma$, and $\theta$, though in most instances the results will be similar. Equation 7 motivates this estimator based on normal theory likelihood; another interpretation of this estimation scheme in terms of a regression using squared residuals as response is described in Davidian and Haaland (1990).

One criticism of estimation based on Equation 7 is that it takes no account of the loss of degrees of freedom due to preliminary estimation of $\beta_{i}$. An alternative is to replace Equation 7 by a suitably modified objective function, as may be derived using the ideas 
underlying restricted maximum likelihood, described by Carroll and Ruppert (1988, p. 7376). The restricted maximum likelihood (REML) objective function is

$$
R E M L_{i}\left(\beta_{i}^{p}, \sigma, \theta\right)=P L_{i}\left(\beta_{i}^{p}, \sigma, \theta\right)-p \log \sigma^{2}+\log \operatorname{det}\left\{\left(X_{i}^{p}\right)^{\prime}\left(G_{i}^{p}\right)^{-1}\left(X_{i}^{p}\right)\right\},
$$

where $X_{i}^{p}$ is the ( $\left.m_{i} \times p\right)$ matrix of derivatives of $f\left(x_{i j}, \beta_{i}\right)$ with respect to $\beta_{i}$ evaluated at $\beta_{i}$ and $\left.G_{i}^{p}=\operatorname{diag}\left[g^{2}\left\{f\left(x_{i j}, \beta_{i}\right)\right\}, \theta\right\}\right]$. The estimator defined by Equation 8 also has a regression interpretation; see Davidian and Carroll (1987).

Note that Equations 7 and 8 base estimation of the variance parameters on squared residuals, which may be expected to give good results for normal errors. It may not be desirable to assume that the data are normally distributed; to allow for the possibility of a heavier-tailed distribution of the $\epsilon_{i j}$, one can replace the role of squared residuals by absolute residuals. Analogous to Equation 7, the absolute residuals (AR) method minimizes in $\eta$ and $\theta$

$$
A R_{i}\left(\beta_{i}^{p}, \sigma, \theta\right)=\sum_{j=1}^{m_{i}}\left(\left|y_{i j}-f\left(x_{i j}, \beta_{i}^{p}\right)\right| /\left[\eta g\left\{f\left(x_{i j}, \beta_{i}^{p}\right), \theta\right\}\right]+\log \left[\eta g\left\{f\left(x_{i j}, \beta_{i}^{p}\right), \theta\right\}\right]\right),
$$

where the role of $\sigma$ is replaced by $\eta=\sigma E\left|\epsilon_{i j}\right|$. Note that Equation 9 can be motivated either by considering a suitable double exponential distribution or by an appropriate regression interpretation (Davidian and Haaland, 1990). As noted by Davidian and Carroll (1987) for individual data, use of Equation 9 can be quite competitive relative to Equations 7 or 8 for estimation of $\theta$ for normal data and significantly better even if only a few outliers are present.

None of the three estimation methods described above can be expected to yield a good characterization of the intra-assay variance when based on data from only a single assay run. Fortunately, it is a straightforward matter to extend these estimation methods to the situation where data are available from a number of assay runs:

1. Obtain, in $n$ separate regressions, preliminary unweighted estimates $\beta_{i}^{p}$ for each assay run.

2. Estimate $\sigma$ and $\theta$ by $\hat{\sigma}$ and $\hat{\theta}$ chosen to minimize $\Sigma O_{i}\left(\beta_{i}^{p}, \sigma, \theta\right)$, where $O_{i}$ corresponds to one of the three objective functions $P L_{i}, R E M L_{i}$, or $A R_{i}$ discussed above.

3. Form estimated weights $\left.g^{-2}\left\{f\left(x_{i j}, \beta_{i}^{p}\right)\right\}, \hat{\theta}\right\}$ and re-estimate the $\beta_{i}$ by $n$ weighted regressions. Treating the results as new preliminary estimates $\beta_{i}^{p}$, return to step 2 . 
It is essential to iterate this scheme to allow the effects of a potentially inefficient initial choice of $\beta_{i}^{p}$ to wash out; experience with assay data suggests that convergence typically occurs between about 4 and 10 iterations.

Computational strategies for point estimation of the variance parameters $(\sigma, \theta)$ by minimizing one of the objective functions in Equations 7-9 are discussed in Davidian and Giltinan (1992b). In the most common special case of the power of the mean variance model (Equation 4), estimation may be recast as a nonlinear least squares problem and thus implemented in any standard statistical software package. Giltinan and Ruppert (1989) present details using PROC NLIN in the SAS software system (SAS User's Guide, Statistics).

An approximate confidence interval for $\theta$ may be constructed using standard profile likelihood methods as follows. Note that $P L_{i}\left(\beta_{i}, \sigma, \theta\right)$ is minus twice the loglikelihood. Substitute the values $\beta_{i}^{p}$ and $\hat{\sigma}$ from the final GLS iteration and define the function

$$
L_{i}(\theta)=-0.5 P L_{i}\left(\beta_{i}^{p}, \hat{\sigma}, \theta\right)
$$

$L_{i}(\theta)$ represents the profile pseudologlikelihood for $\theta$ and is maximized by the pseudolikelihood estimate $\hat{\theta}_{P L}$. An approximate $100(1-\alpha) \%$ confidence interval for $\theta$ is given by

$$
\left\{\theta: L_{i}(\theta) \geq L_{i}\left(\hat{\theta}_{P L}\right)-0.5 \chi^{2}(1,1-\alpha)\right\},
$$

where $\chi^{2}(1,1-\alpha)$ is the $(1-\alpha)$ quantile of the chi-square distribution with 1 degree of freedom. The confidence interval is usually constructed graphically when $\theta$ is a scalar. Development of a confidence interval for the pooled estimation case is analogous, with $L(\theta)$, the profile pseudologlikelihood for the data, being defined as

$$
L(\theta)=\sum_{i=1}^{n} L_{i}(\theta)
$$

We have devoted considerable attention to variance function estimation; we now apply the methods to the DNase and relaxin assay data. In this Section and subsequent Sections, the four-parameter logistic function (Equation 1) was assumed for $f$, and the power of the mean variance model (Equation 4) was assumed for $g$. For both assays, Table 3 summarizes results of the three methods for estimating $\sigma$ and the power parameter $\theta$, both separately by assay and pooled across assays.

For the DNase data, all three methods give similar results in the pooled case, consistent with a $\theta$ value of about 0.5 . If we were to based estimation of $\theta$ on data from individual assays, 
apparent values as high as 1.0 and greater are observed. As discussed in Section 2, and as indicated in Figure 3, considerably different precision profiles may be generated, depending on whether individual-assay or pooled estimates of $\theta$ are substituted in Equation 6.

For relaxin, individual assay estimates of $\theta$ appeared reasonably consistent; the pooled estimate of 1.03 suggests that choice of a default value of $\theta=1.0$ for future work may be reasonable. This analysis addresses the issue of which of the three precision profiles illustrated in Figure 3 is most accurate; that for $\theta=1.0$ is preferred.

Figure 4 shows the precision profiles for run \#1 of the relaxin bioassay; the dotted line is the profile for the value $\theta=1.0$, assuming $r=3$ replicates for the unknown sample. This type of intra-assay precision profile has two main uses in practice. It provides a useful tool for investigating the effect on precision of changing the degree of replication; for instance, to evaluate the gain in precision by increasing replication to $r=4$ for this assay, one can simply regenerate the precision profile using a value of $r=4$ in equation 6 ; this is also shown in Figure 4 (solid line). A second use for the precision profile is as an objective criterion for determining the "usable" portion of the assay range. For instance, for the relaxin bioassay, an intra-assay coefficient of variation of $20 \%$ or less is considered acceptable by users. This leads to the assay ranges shown in Table 4 (based on separate precision profiles for each assay run, assuming $\theta=1.0$ and $r=3$ in all cases). The rule of thumb which had been in effect before formal precision analysis was to take the EC20 to EC80 as the assay range. Inspection of the precision profile shows this rule to be flawed; its symmetry about the inflection point fails to account for the asymmetric nature of the response variability across the response range. The assay range derived from the precision profile, in contrast, is quite asymmetric about the EC50 value, typically corresponding to approximate EC3 to EC55 values. Given the extreme heteroscedasticity in the response, this represents a more credible assessment of the acceptable range.

Figure 4 also shows a precision profile for run \#1 of the relaxin bioassay, assuming $\theta=1.0$ and $r=3$, but ignoring the second term in Equation 6, the error due to fit (dashed line). Comparison with the profile which correctly incorporates both terms in Equation 6 (dotted line) shows that ignoring the error in fitting the standard curve can lead to an overly optimistic assessment of precision.

The idea that pooled estimation of $\theta$ is superior to obtaining estimates based on data from a single assay only is implicit throughout our discussion so far. This belief is vindicated 
by comparison of confidence intervals constructed for pooled and individual estimates of $\theta$. Figures $5(\mathrm{a})$ and (b) display the profile loglikelihood and associated $95 \%$ confidence interval for $\theta$, based on (a) run \#2 of the relaxin assay and (b) pooled data from all nine runs of the bioassay. Pooling information across assays reduces the width of the confidence interval considerably.

As a final illustration of the need for good characterization of the pattern of intraindividual variance, we consider estimation of the minimum detectable concentration (MDC) for these assays. We use the definition of MDC of Rodbard (1978). Let $\bar{y}(x, r)$ be the mean response of $r$ future replicates at concentration $x$ and let $\hat{\beta}_{i}$ be an estimator of $\beta_{i}$. The MDC $x_{M D C}$ at level $(1-\alpha)$ for the ith assay is the smallest value $x$ for which $\operatorname{Pr}\left\{\bar{y}(x, r) \geq f\left(0, \hat{\beta}_{i}\right)\right\} \geq 1-\alpha$. If $t_{\alpha, d f}$, is the $100(1-\alpha)$ percentile of the $t$ distribution with $d f$ degrees of freedom, the usual estimate $\hat{x}_{M D C}$ satisfies

$$
\left\{f\left(\hat{x}_{M D C}, \hat{\beta}_{i}\right)-f\left(0, \hat{\beta}_{i}\right)\right\}^{2}=t_{\alpha, d f}^{2}\left[\hat{\sigma}^{2} g^{2}\left\{f\left(\hat{x}_{M D C}, \hat{\beta}_{i}\right), \theta\right\} / r+v\left\{f\left(0, \hat{\beta}_{i}\right)\right\}\right],
$$

where $v\left\{f\left(0, \hat{\beta}_{i}\right)\right\}$ estimates $\operatorname{Var}\left\{f\left(0, \hat{\beta}_{i}\right)\right\}$ and $\hat{\sigma}^{2}$ is the mean squared error from the weighted fit. A usual choice for $v\left\{f\left(0, \hat{\beta}_{i}\right)\right\}$ would be to substitute $x=0$ in the expression $m_{i}^{-1} f_{\beta}^{\prime}\left(x, \hat{\beta}_{i}\right) \operatorname{Cov}\left\{m_{i}^{-1}\left(\hat{\beta}_{i}-\beta_{i}\right)\right\} f_{\beta}\left(x, \hat{\beta}_{i}\right)$, where $f_{\beta}\left(x, \hat{\beta}_{i}\right)$ is the $\left(m_{i} \times p\right)$ matrix of partial derivatives of $f$ with respect to $\beta_{i}$ evaluated at $\hat{\beta}_{i}$ and $\operatorname{Cov}\left\{m_{i}^{-1}\left(\hat{\beta}_{i}-\beta_{i}\right)\right\}$ is based on the asymptotic normal theory for $\hat{\beta}_{i}$. The value for degrees of freedom $d f$ is based on the degrees of freedom associated with estimation of $\sigma^{2}$; for MDC estimation based on data from the ith assay only, $d f=m_{i}-p$. For MDC estimation using $(\hat{\sigma}, \hat{\theta})$ based on pooled data, $d f=N-n p$; this value will usually be sufficiently large to justify use of the $100(1-\alpha)$ percentile of the standard normal distribution.

Several authors have noted that MDC estimates are conservative if heteroscedasticity in the response is ignored (Oppenheimer, et al., 1983; Davidian, Carroll, and Smith 1988). In the notation of our model, with the power of the mean variance function, this amounts to grossly underestimating $\theta$ (by setting it to zero), which results in overestimation of the response variation at low concentrations. The converse is also true, that overestimation of $\theta$ will lead to underestimation of the response variability at concentrations near zero, with a correspondingly optimistic estimate of the MDC.

To illustrate this point, consider estimation of the MDC for DNase assay \#3. Use of the value of $\theta$ obtained from that assay alone, 1.10 by $P L_{i}$, yields an MDC estimate of 0.0068 
$\mathrm{ng} / \mathrm{ml}$. In contrast, using the pooled estimate of $\theta(0.5)$ results in an MDC estimate of 0.0190 $\mathrm{ng} / \mathrm{ml}$. This discrepancy once again exemplifies the difference in evaluation of precision that can result from pooled versus individual-assay estimation of $\theta$. For the reasons given above, we believe pooled estimation methods to result in superior inference.

\section{Calibration Intervals}

Given an estimated concentration $\hat{x}_{0}$ for an unknown sample from the ith assay run and its estimated standard error $S E\left(\hat{x}_{0}\right)$, a natural question is how one should construct a confidence interval about the calibrated value $\hat{x}_{0}$. We have seen in the previous section that correct evaluation of the precision of a calibrated concentration $\hat{x}_{0}$ is highly dependent on accurate assessment of the variance function for the response, $g\{f(x, \beta), \theta\}$. This is a prerequisite for the formation of accurate calibration confidence intervals. It may not be sufficient, however, as the following development shows. In contrast to the case of inverse linear regression, the literature on nonlinear regression does not offer much guidance on the formation of inverse prediction intervals. In this section, we compare the performance of three different techniques for the DNase and relaxin data sets. The methods described below by no means exhaust the possible approaches to the problem. We consider the following three methods in detail here:

1. Wald intervals on the untransformed concentration scale. Form the confidence interval according to the formula

$$
\hat{x}_{0} \pm z_{1-\alpha / 2} S E\left(\hat{x}_{0}\right)
$$

where $S E\left(\hat{x}_{0}\right)$ is the square root of the estimated variance of $\hat{x}_{0}$, calculated according to the formula in Equation 6, where $\left(\beta_{i}, \sigma, 0\right)$ are estimated by one of the pooled GLS schemes of the previous section, and $z_{1-\alpha / 2}$ represents the $100(1-\alpha / 2)$ th percentile of the standard normal distribution. The rationale for using a critical value from $z-$ rather than from $t$ - tables is the accumulation of degrees of freedom in the pooled estimation of $(\sigma, \theta)$ from a series of assays.

2. Wald intervals on the log concentration scale. By definition, the intervals above are symmetric on the concentration scale. Typically, standard concentrations are chosen according to a geometric dilution series, so that a calibration interval which is symmetric on the log scale might seem more natural. A supporting argument for this approach is that exact, Fieller-type, inverse prediction intervals obtained in the linear 
bioassay case are more nearly symmetric on the log scale than on the untransformed concentration scale. This suggests forming a calibration interval by exponentiating the limits

$$
\log \hat{x}_{0} \pm z_{1-\alpha / 2} S E\left(\log \hat{x}_{0}\right),
$$

where $\mathrm{SE}\left(\log \hat{x}_{0}\right)$ is obtained by standard Taylor series methods, with due incorporation of the presumed heteroscedasticity in the response.

3. Invert the asymptotic prediction interval. Using this approach, the calibration interval for $\hat{x}_{0}$ is obtained by inverting the limits of the prediction interval around $y_{0}$. More explicitly, a $100(1-\alpha) \%$ confidence interval for $x_{0}$ is given by

$$
I=\left\{x \text { such that } y_{0} \in I(x)\right\}
$$

where $I(x)=\left\{f\left(x, \hat{\beta}_{i}\right) \pm z_{1-\alpha / 2} S E\left\{f\left(x, \hat{\beta}_{i}\right)\right\}\right.$ and $S E\left\{f\left(x, \hat{\beta}_{i}\right)\right\}=\left[\hat{\sigma}^{2} g^{2}\left\{f\left(x, \hat{\beta}_{i}\right), \theta\right\}+\right.$ $\left.m_{i}^{-1} f_{\beta}^{\prime}\left(x, \hat{\beta}_{i}\right) \operatorname{Cov}\left\{m_{i}^{-1}\left(\hat{\beta}_{i}-\beta_{i}\right)\right\} f_{\beta}\left(x, \hat{\beta}_{i}\right)\right]$. Note that this interval, formed by inverting one which is symmetric on the response scale, is not, in general, symmetric on the concentration or log concentration scale, particularly at concentrations where the response is close to the upper or lower limits.

Each of these methods of forming confidence intervals is based on a large-sample approximation. There is no reason a priori to know which method is best, or indeed if any of the three techniques exhibits uniform superiority in terms of coverage probability. In fact, it is not guaranteed that any of the methods will necessarily achieve the nominal level in general. It is thus of considerable interest to see how the methods compare in practice.

Figure $6(\mathrm{a})$ shows $95 \%$ confidence intervals constructed by each of the three methods for run \#2 of the DNase assay, based on $r=2$ replicates of the response. For each method, $\left(\beta_{i}, \sigma, \theta\right)$ used to construct the interval were obtained from the pooled GLS scheme with the pseudolikelihood objective function. All three methods give virtually identical results. For run \#2 of the relaxin assay, Figure 6 (b) shows the three types of $95 \%$ confidence intervals based on $r=3$ replicates; in contrast, the picture is quite different. There is reasonable agreement among the methods in the middle portion of the concentration range, but the results diverge tremendously at the extreme concentrations. This lack of agreement among methods relying on standard approximations is disconcerting. It is unclear for the data at hand which method yields the most accurate results. Simulation work and theoretical 
investigation are needed to resolve this issue; this work is in progress, but goes beyond the scope of this chapter.

\section{Bayesian Calibration}

The development and examples in Section 3 emphasized the advantages to be gained by estimating variance parameters based upon pooled data across several assay runs rather than data from only a single run. Given the hierarchical nature of the model for the regression parameters, it is natural to ask whether similar gains can be achieved in estimating the $\beta_{i}$ by pooling data across assay runs in an appropriate Bayesian fashion. While implementation of Bayesian techniques is common in the population pharmacokinetic literature (Vozeh, et al. 1985), they are rarely used in the calibration context; an exception is the paper by Unadkat, et al. (1986). In this section, we describe one method for Bayesian calibration and investigate its performance for the relaxin data and by limited simulation work.

In the calibration context, enough concentration-response data are generally available for the standard to allow individual estimates $\beta_{i}^{*}$ to be obtained for the regression parameters. In the numerical illustrations below we consider the specific case where $\beta_{i}^{*}$ is a GLS estimate with weights based on the pooled pseudolikelihood estimates of $(\sigma, \theta)$ assuming the power of the mean variance model of Equation 4. For any individual estimator $\beta_{i}^{*}$, a refined estimate incorporating shrinkage to the mean, $\beta$, may be derived based on the assumption that

$$
\beta_{i}^{*} \mid \beta_{i} \sim N\left(\beta_{i}, V_{i}\right)
$$

holds, at least approximately, where $V_{i}$ is an estimate of the asymptotic covariance matrix. This assumption implies that

$$
\beta_{i}^{*} \sim N\left(\beta, V_{i}+\Sigma\right)
$$

under normality of the $\beta_{i}$, which suggests maximum likelihood estimation of the population values $\beta$ and $\Sigma$ as a feasible approach. One method of accomplishing this is by an iterative EM algorithm referred to by Steimer, et al. (1984) as the Global Two-Stage Method (GTS). At iteration $(\mathrm{k}+1)$ :

1. Produce refined estimates of $\beta_{i}$ :

$$
\left(\hat{\beta}_{i}\right)_{(k+1)}=\left(V_{i}^{-1}+\Sigma_{(k)}^{-1}\right)^{-1}\left(V_{i}^{-1} \beta_{i}^{*}+\Sigma_{(k)}^{-1} \hat{\beta}_{(k)}\right), i=1, \ldots, n
$$


2. Produce updated estimates of the population parameters:

$$
\begin{gathered}
\hat{\beta}_{(k+1)}=n^{-1} \sum_{i=1}^{n}\left(\hat{\beta}_{i}\right)_{(k+1)}, \\
\hat{\Sigma}_{(k+1)}=n^{-1} \sum_{i=1}^{n}\left\{\left(\hat{\beta}_{i}\right)_{(k+1)}-\hat{\beta}_{(k+1)}\right\}\left\{\left(\hat{\beta}_{i}\right)_{(k+1)}-\hat{\beta}_{(k+1)}\right\}^{\prime}+n^{-1} \sum_{i=1}^{n}\left(V_{i}^{-1}+\Sigma_{(k)}^{-1}\right)^{-1} .
\end{gathered}
$$

Reasonable starting values are provided by

$$
\hat{\beta}_{(0)}=n^{-1} \sum_{i=1}^{n} \beta_{i}^{*} ; \hat{\Sigma}_{(0)}=n^{-1} \sum_{i=1}^{n}\left(\beta_{i}^{*}-\hat{\beta}_{(0)}\right)\left(\beta_{i}^{*}-\hat{\beta}_{(0)}\right)^{\prime},
$$

and $\beta_{i}^{*}$ and $V_{i}$ remain fixed throughout. A similar method derived from a hierarchical Bayesian perspective has been developed by Racine-Poon (1985). The refined individual estimates in the first step are approximate current Bayes estimates, borrowing information across the sample.

Table 5 summarizes results of the GTS scheme for the relaxin bioassay data. GLS regression parameter estimates, $\beta_{i}^{*}$, obtained for each assay using the pooled pseudolikelihood estimates of $\sigma$ and $\theta$ and the refined GTS versions of these estimates, $\hat{\beta}_{i}$, are presented in Table 5(a). To illustrate the effect of the Bayesian refinement on calibration, Table 5(b) shows calibrated concentrations for responses at several levels based on the unrefined estimates $\beta_{i}^{*}$ and refined estimates $\hat{\beta}_{i}$ for assay \#9. Differences of up to $15 \%$ of calibrated values are observed.

To investigate this issue more closely, we performed simulations to examine the effect of different methods of estimation of the standard curve for a particular experiment on subsequent calibration inference for that experiment. We report the results of a typical study here. The simulation scenario was as follows. Ten individual parameters $\beta_{i}$ were generated from a $N(\beta, \Sigma)$ distribution with $\beta=[2.31,0.10,1.40,1.00]^{\prime}$ and $\Sigma$ given by

$$
\left(\begin{array}{cccc}
0.02 & 0.0002 & 0.0015 & -0.001 \\
0.0002 & 0.00005 & -0.0002 & 0.0002 \\
0.0015 & -0.0002 & 0.002 & -0.001 \\
-0.001 & 0.0002 & -0.001 & 0.0016
\end{array}\right)
$$

These $\beta_{i}$ values were regarded as coming from a series of fixed assay experiments and remained fixed for the simulation. 50 data sets were generated, each consisting of duplicate observations for each experiment at doses $x=0.0,1.5625,3.125,6.25$, and 12.5 according 
to Equation 2 with $\epsilon_{i}$ with distribution $N\left(0, I_{m_{i}}\right)$, power of the mean variance function, $(\sigma, \theta)=(0.08,0.8)$, and mean response given by the four-parameter logistic function evaluated at $\left(x, \beta_{i}\right)$. GTS methods were applied to each data set with the following possiblities for $\beta_{i}^{*}$ at stage 1:

1. OLS (assume $\theta=0.0$ )

2. GLS assuming $\theta$ known and equal to the true value of 0.8

3. GLS with PL estimation of $(\sigma, \theta)$ pooled across all 10 experiments

4. GLS with REML estimation of $(\sigma, \theta)$ pooled across all 10 experiments

5. GLS with AR estimation of $(\sigma, \theta)$ pooled across all 10 experiments

6. GLS with PL estimation of $(\sigma, \theta)$ separately for each experiment

7. GLS with REML estimation of $(\sigma, 0)$ separately for each experiment

8. GLS with AR estimation of $(\sigma, \theta)$ separately for each experiment

The $\beta_{i}^{*}$ for all cases were input to GTS and final "refined" estimates $\hat{\beta}_{i}$ obtained. Our interest focused on the quality of estimation of $\beta_{10}$, the regression parameter for the tenth experiment, by $\beta_{10}^{*}$ and the refined estimate $\hat{\beta}_{10}$, and on the quality of calibration inference based on $\beta_{10}^{*}$ and $\hat{\beta}_{10}$ for each of the eight estimation schemes. For calibration, for each simulation data set, an observation $y_{0}$ was generated from the experiment at dose $x_{0}=0.30$. Estimates of $x_{0}$ were obtained for each method based on both $\beta_{10}^{*}$ and $\hat{\beta}_{10}$ by inverse regression, that is, substitution into Equation 5.

Results for the three objective functions used to estimate $\theta$ were qualitatively similar in all cases. Accordingly, in Tables 6(a) and (b) we include results only for the pseudolikelihood methods. In Table 6(a), mean square error (MSE) ratios are given to compare estimation of the elements of $\beta_{10}$ by $\beta_{10}^{*}$ and $\hat{\beta}_{10}$ for each method. In all cases, a marked increase in efficiency is realized by using refined estimates both for parameter estimation and for calibration, reflecting the standard Bayesian vicw that one may gain efficiency for estimation for any single assay by borrowing information across assays. Accordingly, in Table 6(b), results are given for refined estimates only. This Table presents MSE ratios to compare the estimates of elements of $\beta_{10}$ and $x_{0}$ when $\theta$ is incorrectly specified (OLS) or estimated 
(GLS with PL estimation of $\theta$ based on pooled and individual assay data) to those obtained when $\theta$ is correctly assumed to be equal to the true value 0.8 . The quality of estimation deteriorates when based on OLS, reflecting the well-established need for weighting in assay analysis, particularly for calibration. Estimation is also of poorer quality if $\theta$ is estimated separately for each experiment, but performance equal to that attained when $\theta=0.8$ is known is possible if information on intra-assay variance is pooled to estimate $\theta$.

These results suggest that as long as the pattern of intra-assay variability is similar across experiments, characterization of a particular standard curve and subsequent calibration inference will be improved if information is pooled across previous experiments, both for the regression parameters in the Bayesian sense and for characterization of intra-assay variance.

\section{Discussion}

We have used data from the DNase immunoassay and the NHE cell bioassay for relaxin to examine several issues which arise in the calibration of recombinant polypeptides intended for therapeutic use, such as hormones, enzymes, or monoclonal antibodies. The data chosen for inclusion in this case study are representative of their assay types. The DNase data are reasonably "well-behaved," attaining a degree of intra- and inter-assay precision that is fairly typical for immunoassays. In contrast, the relaxin data exhibit a much greater degree of variability, both within and across assays. Our experience has been that this level of variability is quite common in bioassay data.

Several conclusions may be drawn from our examination of these data sets:

- A useful general framework for calibration inference is provided by a nonlinear mixed effects model which allows for heteroscedasticity of intra-assay response, as described in Section 2. The four-parameter logistic function and the power variance function represent the most common model specifications within this framework, but other model choices may be substituted where appropriate.

- It is important to characterize the pattern of intra-assay variability correctly; within the parametric framework of Section 2, this corresponds to a correct assessment of the variance parameters $(\sigma, \theta)$. In general, one cannot expect to achieve this goal based on data from only a single assay run. The methods described in Section 3 show how estimation of the intra-assay variance parameters can be accomplished based on data 
from several runs. Our treatment of this topic is in no way exhaustive. We have focused on methods based on residuals from the fitted regressions. For a discussion of other methods, in particular, those which take advantage of replication in the response, the reader is referred to Carroll and Ruppert (1988, chap. 3).

- Improved estimation of intra-assay variance parameters pays off in several ways. It allows construction of accurate intra-assay precision profiles which may be used to investigate issues such as the appropriate degree of replication for standards and unknown samples, as well as to provide an objective criterion for setting the assay range. In addition, correct estimation of $(\sigma, \theta)$ helps in the reliable evaluation of assay constructs such as the MDC, as well as being an essential prerequisite for the formation of prediction or calibration intervals.

Our examination of the DNase and relaxin assay data also raises some issues which have not been completely resolved in this chapter:

- The reader has been alerted to the fact that, in constructing calibration intervals, three "standard" approximations, described in Section 4, can give widely divergent results. Our suspicion, based on empirical evidence provide by other assays, is that the results displayed in Figures 6(a) and 6(b) are representative, in the sense that the method of constructing confidence intervals makes little difference for well-behaved (e.g., immunoassay) data, but certainly matters for highly variable assays like the relaxin bioassay. This question can properly be assessed by appropriate simulation work. We are in the process of examining the performance of these and other methods by Monte Carlo work; this investigation goes beyond the scope of this case study, however.

- To our knowledge, Bayesian calibration methods of the type proposed in Section 5 are not widely used in practice. The simulation evidence (Table 6) suggests a considerable potential gain in efficiency. However, this gain must be weighed against the practicality of implementing such a calibration scheme in an environment where hundreds of thousands of samples are processed in several hundred assays annually, typically the case for an established biotechnology company. Furthermore, regulatory issues may mitigate against routine use of Bayesian calibration methods; where regulatory requirements stipulate strict guidelines for sample tracking, audit trails, and software 
validation, the practical drawbacks to routine implementation of Bayesian calibration may outweigh potential benefits. We note in this context also that the techniques for pooled estimation of intra-assay variation proposed in Section 3 are most likely to be of use during the assay development stage, rather than once an assay is in "production" mode.

No case study can hope to address all the issues in assay development and calibration. Several topics of practical importance not covered in our discussion are:

- Design issues, such as the choice and spacing of standard concentrations; physical layout of standards and samples on the microtiter plates used in these assays.

- Assessment of variability other than intra-plate variation, for example, interplate within day and interday variation; placement of control samples to investigate this; estimation of variance components in the presence of heteroscedasticity in one or more component.

- Assay "quality control", such as tracking assay performance, setting acceptability criteria, guidelines for resampling.

Our experience has been that treatment of the issues discussed in this case study has been scattered throughout the statistical and clinical chemistry literature, when it exists at all. We hope that the unified presentation in this chapter proves useful both to biostatisticians and assay practitioners dealing with issues arising in nonlinear calibration.

\section{REFERENCES}

Beal, S. L. and Sheiner, L. B. (1982). Estimating population kinetics. CRC Critical Reviews in Biomedical Engineering 8, 195-222.

Carroll, R. J. and Ruppert, D. (1982). A comparison between maximum likelihood and generalized least squares in a heteroscedastic linear model. Journal of the American Statistical Association 77, 878--882.

Carroll, R. J. and Ruppert, D. (1988). Transformation and Weighting in Regression. New York: Chapman and Hall. 
Clark, B. C. and Engvall, E. (1980). Enzyme linked immunopsorbent assay (ELISA): theoretical and practical aspects. Enzyme-immunoassay. Ed. E. T. Maggio, Boca Raton: CRC Press.

Davidian, M. and Carroll, R. J. (1987). Variance function estimation. Journal of the American Statistical Association 82, 1079-1091.

Davidian, M., Carroll, R. J. and Smith, W. (1988). Variance functions and the minimum detectable concentration in assays. Biometrika 75, 549-556.

Davidian, M. and Giltinan, D. M. (1992a). Some estimation methods for nonlinear mixed effects models. Journal of Biopharmaceutical Statistics, to appear.

Davidian, M. and Giltinan, D. M. (1992b). Some simple methods for estimating intraindividual variability in nonlinear mixed effects models. Biometrics, in press.

Davidian, M. and Haaland, P. D. (1990). Regression and calibration with nonconstant variance. Chemometrics and Intelligent Laboratory Systems 9, 231-248

Ekins, R. P. and Edwards, P. R. (1983). The precision profile: its use in assay design, assessment, and quality control. Immunoassays for Clinical Chemistry, Eds. W. M. Hunter and J. E. T. Corrie, Edinburgh: Churchill Livingston.

Fei, D. T. W., Gross, M. C., Lofgren, J., Mora-Worms, M. and Chen, A. B. (1990). Cyclic AMP response to recombinant human relaxin by cultured human endometrial cells-a specific and high throughput in-vitro hioassay. Biochemical and Biophysical Research Communications 170, 214-222.

Finney, D. J. (1976). Radioligand assay. Biometrics 32, 721-740.

Giltinan, D. M. and Ruppert, D. (1989). Fitting heteroscedastic regression models to individual pharmacokinetic data using standard statistical software. Journal of Pharmacokinetics and Biopharmaceutics 17, 601-614.

Oppenheimer, L., Capizzi, T. P., Weppelman, R. M. and Mehta, H. (1983). Determining the lowest limit of reliable assay measurement. Analytical Chemistry 55, 638-643. 
Peck, C. C., Beal, S. L., Sheiner, L. B. and Nichols, A. I. (1984). Extended least squares nonlinear regression: a possible solution to the choice of weights problem in analysis of individual pharmacokinetic data. Journal of Pharmacokinetics and Biopharmaceutics $12,545-558$.

Racine-Poon, A. (1985). A Bayesian approach to nonlinear random effects models. Biometrics $41,1015-1023$.

Rodbard, D. and Frazier, G. R. (1975). Statistical analysis of radioligand assay data. Methods of Enzymology 37, 839-841.

Statistical Analysis System User's Guide, Statislics, Version 6.0. SAS Institute, Cary NC.

Steimer, J. L., Mallet, A., Golmard, J. L. and Boisvieux, J. F. (1984). Alternative approaches to estimation of population pharmacokinetic parameters: comparison with the nonlinear mixed effect model. Drug Metabolism Review 15, 265-292.

Unadkat, J. D., Beal, S. L. and Sheiner, J. B.(1986). Bayesian calibration. Analytica Chimica Acta 181, 27-86.

van Houwelingen, J. C. (1988). Use and abuse of variance models in regression. Biometrics 43, 1073-1081.

Vozeh, S., Hillman, R., Wandell, M., Ludden, T. M., and Sheiner, L. B. (1985). Computerassisted drug assay interpretation based on Bayesian estimation of individual pharamacokinetics: application to lidocaine. Therapeutic Drug Monitoring 7, 66-73. 
Table 1. DNase ELISA assay responses for eleven experiments; $x=D N a s e$ concentration $(\mathrm{ng} / \mathrm{ml}), y=a b s o r b a n c e$.

\begin{tabular}{|c|c|c|c|c|}
\hline$x$ & 1 & 2 & 3 & 4 \\
\hline $\begin{array}{l}0.00 \\
0.1953125 \\
0.390625 \\
0.78125 \\
1.5625 \\
3.125 \\
6.25 \\
12.5\end{array}$ & $\begin{array}{l}0.047,0.057 \\
0.159,0.155 \\
0.246,0.252 \\
0.427,0.411 \\
0.704,0.684 \\
0.994,0.980 \\
1.421,1.385 \\
1.715,1.721\end{array}$ & $\begin{array}{l}0.045,0.050 \\
0.137,0.123 \\
0.225,0.207 \\
0.401,0.383 \\
0.672,0.681 \\
1.116,1.078 \\
1.554,1.526 \\
1.932,1.914\end{array}$ & $\begin{array}{l}0.070,0.068 \\
0.173,0.165 \\
0.277,0.248 \\
0.434,0.426 \\
0.703,0.689 \\
1.067,1.077 \\
1.629,1.479 \\
2.003,1.884\end{array}$ & $\begin{array}{l}0.011,0.016 \\
0.118,0.108 \\
0.200,0.206 \\
0.364,0.360 \\
0.620,0.640 \\
0.979,0.973 \\
1.424,1.399 \\
1.740,1.732\end{array}$ \\
\hline$x$ & 5 & 6 & 7 & 8 \\
\hline $\begin{array}{l}0.00 \\
0.1953125 \\
0.390625 \\
0.78125 \\
1.5625 \\
3.125 \\
6.25 \\
12.5\end{array}$ & $\begin{array}{l}0.035,0.035 \\
0.132,0.135 \\
0.224,0.220 \\
0.385,0.390 \\
0.658,0.647 \\
1.060,1.031 \\
1.425,1.409 \\
1.750,1.738\end{array}$ & $\begin{array}{l}0.086,0.103 \\
0.191,0.189 \\
0.272,0.277 \\
0.440,0.126 \\
0.686,0.676 \\
1.062,1.072 \\
1.424,1.459 \\
1.768,1.806\end{array}$ & $\begin{array}{l}0.094,0.092 \\
0.182,0.182 \\
0.282,0.273 \\
0.444,0.439 \\
0.686,0.668 \\
1.052,1.035 \\
1.409,1.392 \\
1.759,1.739\end{array}$ & $\begin{array}{l}0.054,0.054 \\
0.152,0.148 \\
0.226,0.222 \\
0.392,0.383 \\
0.658,0.644 \\
1.043,1.002 \\
1.466,1.381 \\
1.740,1.724\end{array}$ \\
\hline
\end{tabular}

\begin{tabular}{lccc}
\hline$x$ & 9 & 10 & 11 \\
\hline 0.00 & $0.032,0.043$ & $0.052,0.094$ & $0.017,0.018$ \\
0.1953125 & $0.142,0.155$ & $0.164,0.166$ & $0.121,0.124$ \\
0.390625 & $0.239,0.242$ & $0.259,0.256$ & $0.206,0.215$ \\
0.78125 & $0.420,0.395$ & $0.439,0.439$ & $0.377,0.374$ \\
1.5625 & $0.624,0.705$ & $0.690,0.701$ & $0.614,0.609$ \\
3.125 & $1.046,1.026$ & $1.042,1.075$ & $1.019,1.001$ \\
6.25 & $1.398,1.405$ & $1.340,1.406$ & $1.334,1.364$ \\
12.5 & $1.693,1.729$ & $1.699,1.708$ & $1.730,1.710$ \\
& & & \\
\hline
\end{tabular}


Table 2. Relaxin bioassay responses for nine experiments; $x=$ relaxin concentration $(\mathrm{ng} / \mathrm{ml}), y=c A M P$ concentration (pmoles $/ \mathrm{ml})$.

\begin{tabular}{|c|c|c|c|}
\hline$x$ & 1 & 2 & 3 \\
\hline $\begin{array}{l}0.00 \\
0.34 \\
0.69 \\
1.38 \\
2.75 \\
5.50 \\
11.00 \\
22.00\end{array}$ & $\begin{array}{l}1.77 \\
3.35,4.00,6.10 \\
8.40,12.00,8.15 \\
13.25,24.25,17.85 \\
40.15,49.35,40.05 \\
61.85,85.15,58.80 \\
95.05,118.50,76.25 \\
116.70,140.45,90.40\end{array}$ & $\begin{array}{l}1.80 \\
4.20,5.85,4.70 \\
7.10,9.20,9.00 \\
15.00,23.45,25.40 \\
30.15,46.90,74.45 \\
51.85,72.95,83.70 \\
66.30,101.25,92.80 \\
87.50,115.30,109.40\end{array}$ & $\begin{array}{l}1.87 \\
3.60,4.70,4.20 \\
8.65,8.60,4.85 \\
13.00,16.95,13.85 \\
24.60,34.60,31.70 \\
57.45,58.25,49.05 \\
60.55,77.80,78.95\end{array}$ \\
\hline$x$ & 4 & 5 & 6 \\
\hline $\begin{array}{l}0.00 \\
0.34 \\
0.69 \\
1.38 \\
2.75 \\
5.50 \\
11.00 \\
22.00\end{array}$ & $\begin{array}{l}1.62 \\
3.65,3.60,4.95 \\
5.35,7.35,9.00 \\
10.80,18.25,19.60 \\
21.75,26.05,32.60 \\
57.05,92.05,99.90 \\
85.10,92.30,104.90 \\
87.55,97.35,101.60\end{array}$ & $\begin{array}{l}1.03 \\
1.60,2.30,2.95 \\
3.15,5.05,6.70 \\
7.60,11.55,12.80 \\
14.90,23.75,27.55 \\
34.85,61.50,50.00 \\
47.40,57.20,61.25 \\
38.70,55.30,63.20\end{array}$ & $\begin{array}{l}1.37 \\
3.95,4.00,3.75 \\
6.00,10.60,10.00 \\
16.20,23.95,22.30 \\
34.80,61.75,54.05 \\
62.25,112.90,105.60 \\
99.30,137.15,155.10 \\
101.95,166.30,177.60\end{array}$ \\
\hline$x$ & 7 & 8 & 9 \\
\hline $\begin{array}{l}0.00 \\
0.34 \\
0.69 \\
1.38 \\
2.75 \\
5.50 \\
11.00 \\
22.00\end{array}$ & $\begin{array}{l}1.17 \\
3.15,3.70,3.70 \\
2.45,6.45,6.25 \\
11.85,16.75,23.55 \\
28.45,42.45,49.40 \\
51.75,91.25,77.10 \\
81.10,122.90,125.90 \\
72.90,118.20,117.95\end{array}$ & $\begin{array}{l}1.87 \\
5.40,4.55,3.05 \\
7.35,7.10,7.30 \\
11.10,16.35,14.15 \\
25.70,27.85,35.90 \\
49.30,63.60,72.95 \\
57.50,71.45,69.75 \\
69.65,92.05,88.55\end{array}$ & $\begin{array}{l}2.32 \\
4.85,4.50,4.20 \\
8.40,7.90,7.05 \\
12.90,18.20,16.10 \\
31.25,37.55,35.75 \\
65.95,94.60,71.65 \\
63.75,68.85,82.60 \\
83.70,103.85,93.00\end{array}$ \\
\hline
\end{tabular}


Table 3. Estimates of $(\sigma, \theta)$ by several methods, based on the power of the mean variance model.

(a) DNase assay

(b) Relaxin bioassay

\begin{tabular}{cccccccc} 
Assay run & PL & AR & REML & Assay run & PL & AR & REML \\
& & & & & & & \\
\hline 1 & $0.022,0.549$ & $0.022,0.490$ & $0.022,0.526$ & 1 & $0.269,0.926$ & $0.244,0.955$ & $0.308,0.885$ \\
2 & $0.015,0.384$ & $0.015,0.359$ & $0.015,0.373$ & 2 & $0.143,1.158$ & $0.149,1.146$ & $0.198,1.061$ \\
3 & $0.038,1.101$ & $0.038,1.100$ & $0.037,1.059$ & 3 & $0.206,0.904$ & $0.189,0.933$ & $0.232,0.866$ \\
4 & $0.015,0.427$ & $0.014,0.393$ & $0.015,0.410$ & 4 & $0.156,1.157$ & $0.176,1.120$ & $0.200,1.079$ \\
5 & $0.019,1.260$ & $0.019,1.261$ & $0.017,1.113$ & 5 & $0.268,0.988$ & $0.259,1.000$ & $0.311,0.934$ \\
6 & $0.016,0.385$ & $0.017,0.437$ & $0.016,0.380$ & 6 & $0.098,1.212$ & $0.062,1.353$ & $0.124,1.144$ \\
7 & $0.016,0.776$ & $0.015,0.689$ & $0.015,0.720$ & 7 & $0.345,0.929$ & $0.287,0.989$ & $0.391,0.891$ \\
8 & $0.027,0.927$ & $0.029,1.014$ & $0.026,0.862$ & 8 & $0.216,0.952$ & $0.202,0.973$ & $0.247,0.908$ \\
9 & $0.026,0.446$ & $0.025,0.352$ & $0.026,0.416$ & 9 & $0.033,1.469$ & $0.042,1.399$ & $0.044,1.383$ \\
10 & $0.025,0.182$ & $0.024,0.104$ & $0.025,0.178$ & & & & \\
11 & $0.025,0.955$ & $0.024,0.904$ & $0.024,0.918$ & & & & \\
Pooled & $0.023,0.503$ & $0.024,0.527$ & $0.023,0.486$ & Pooled & $0.204,1.028$ & $0.182,1.065$ & $0.241,0.976$
\end{tabular}

Table 4. Assay reporting ranges for the relaxin bioassay.

Assay run Conc. range $(\mathrm{ng} / \mathrm{ml})$ with $C V<20 \%$ EC20 to EC80 (ng/ml)

\begin{tabular}{lll}
\hline 1 & 0.50 to 4.9 (EC4 to EC50) & 1.7 to 12.6 \\
2 & 0.48 to 3.1 (EC3 to EC52) & 1.7 to 10.7 \\
3 & 0.38 to 6.8 (EC2 to EC 62) & 1.85 to 12.0 \\
4 & 0.65 to 4.0 (EC3 to EC.46) & 2.4 to 6.6 \\
5 & 0.50 to 3.0 (EC3.5 to EC44) & 1.7 to 6.0 \\
6 & 0.30 to 4.9 (EC1 to EC53) & 1.9 to 10.4 \\
7 & 0.60 to 3.6 (EC2 to EC51) & 1.85 to 7.0 \\
8 & 0.50 to 5.4 (EC2.5 to EC64) & 1.75 to 8.0 \\
9 & 0.45 to 5.5 (EC2 to EC70) & 1.7 to 6.5 \\
\hline
\end{tabular}


Table 5. (a) Estimation of $\beta$ based on pooled GLS and refined GTS estimation for the relaxin bioassay. (b) Calibration based on pooled GLS and refined GTS estimation, experiment 9, relaxin bioassay.

\begin{tabular}{cccccccccc}
\hline \multicolumn{1}{c}{ Pooled GLS-PL } & \multicolumn{1}{c}{ (a) } \\
Assay run & $\beta_{1}$ & $\beta_{2}$ & $\beta_{3}$ & $\beta_{4}$ & $\beta_{1}$ & $\beta_{2}$ & $\beta_{3}$ & $\beta_{4}$ \\
\hline & & & & & & & & & \\
\hline & 127.32 & 1.76 & 1.57 & 1.43 & 120.47 & 1.89 & 1.47 & 1.52 \\
2 & 105.56 & 1.90 & 1.23 & 1.59 & 119.60 & 1.94 & 1.47 & 1.51 \\
3 & 101.83 & 1.91 & 1.69 & 1.38 & 91.09 & 1.84 & 1.45 & 1.49 \\
4 & 121.58 & 1.76 & 1.66 & 1.49 & 106.06 & 1.65 & 1.45 & 1.54 \\
5 & 62.13 & 1.06 & 1.30 & 1.64 & 65.72 & 1.16 & 1.40 & 1.59 \\
6 & 164.12 & 1.38 & 1.56 & 1.58 & 145.17 & 1.54 & 1.47 & 1.61 \\
7 & 121.39 & 1.34 & 1.47 & 1.68 & 113.89 & 1.29 & 1.44 & 1.49 \\
8 & 93.83 & 2.02 & 1.56 & 1.44 & 89.89 & 1.85 & 1.44 & 1.49 \\
9 & 98.45 & 2.56 & 1.37 & 1.64 & 103.35 & 1.92 & 1.46 & 1.49 \\
& & & & & & & & & \\
\hline
\end{tabular}

(b)

response level estimated conc. based on GLS-PL estimated conc. based on GTS

\begin{tabular}{lll}
\hline 3.5 & 0.238 & 0.269 \\
4.0 & 0.309 & 0.324 \\
6.5 & 0.579 & 0.558 \\
8.0 & 0.712 & 0.681 \\
20.0 & 1.579 & 1.544 \\
60.0 & 5.038 & 5.219 \\
\hline
\end{tabular}

Table 6. (a) Monte Carlo MSE for refined GTS estimate of $\beta_{10}$ divided by MSE for estimate of $\beta_{10}$ obtained by the method indicated, expressed as a percentage. In each case, the GTS estimate was obtained using the method indicated as input. (b) Monte Carlo MSE for refined GTS estimale of $\beta_{10}$ using PTS $(\theta=0.8)$ as input divided by $M S E$ for refined GTS estimate of $\beta_{10}$ oblained by using the method indicated as input, expressed as a percentage.

(a)

Element, of $\beta_{10}$

$\begin{array}{lllllllllll}\text { Method input to GTS } & \beta_{1} & \beta_{2} & \beta_{3} & \beta_{4} & x_{0} & \beta_{1} & \beta_{2} & \beta_{3} & \beta_{4} & x_{0}\end{array}$

\begin{tabular}{lrrrrrrrrrr}
\hline OLS & 33.7 & 83.2 & 70.7 & 12.5 & 43.4 & 47.9 & 40.5 & 62.9 & 83.3 & 76.5 \\
GLS, $\theta=0.8$ & 18.6 & 41.7 & 37.1 & 40.2 & 37.6 & 100.0 & 100.0 & 100.0 & 100.0 & 100.0 \\
GLS-PL, individual data & 14.4 & 49.3 & 32.7 & 38.8 & 41.9 & 65.8 & 67.3 & 67.0 & 82.0 & 86.4 \\
GLS-PL, pooled data & 13.9 & 62.2 & 38.6 & 47.5 & 37.9 & 118.8 & 84.6 & 111.6 & 105.9 & 100.5 \\
\hline
\end{tabular}


Figure $1:$ ELISA for DNase in Rat Serum

Assay 1

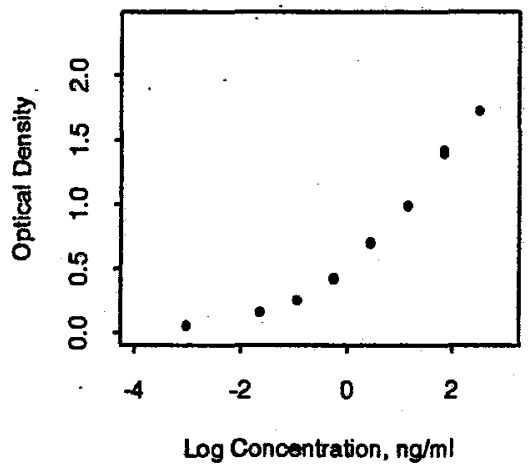

Assay 4

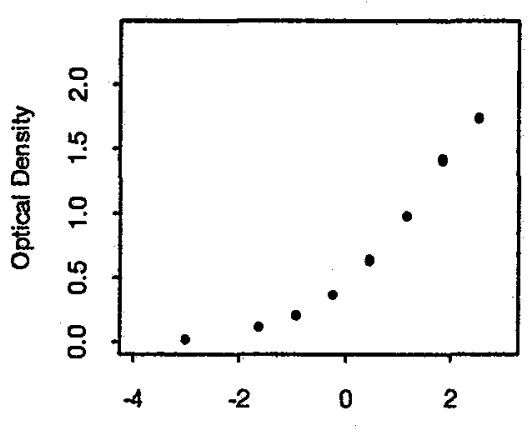

Log Concentration, ng/ml

Assay 7

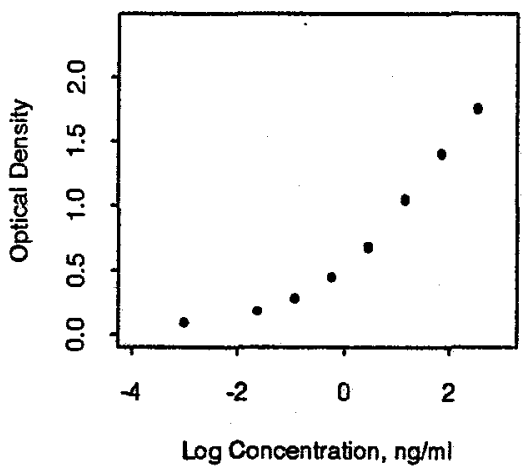

Assay 10

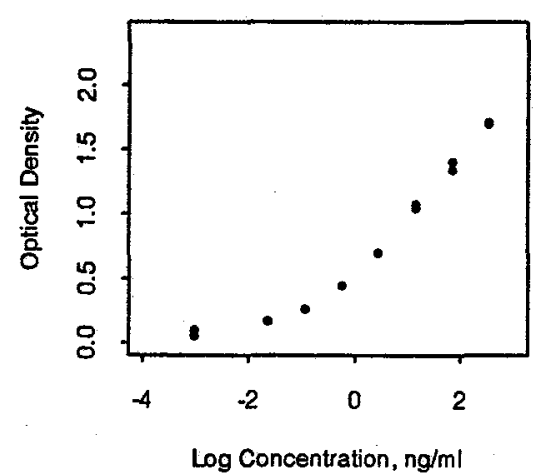

Assay 2

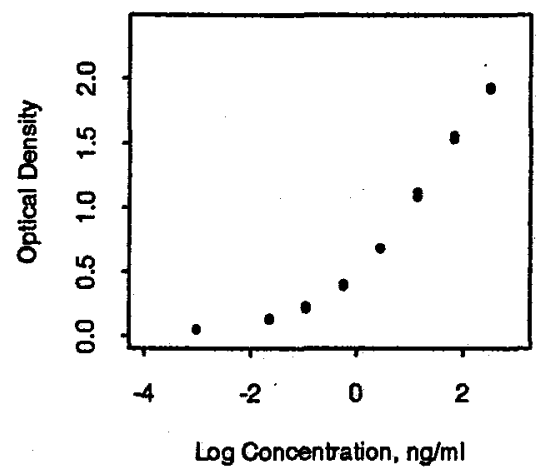

Assay 5

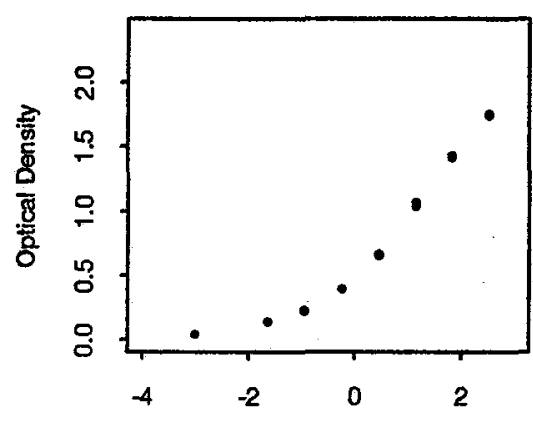

Log Concentration, ng/m!

Assay 8

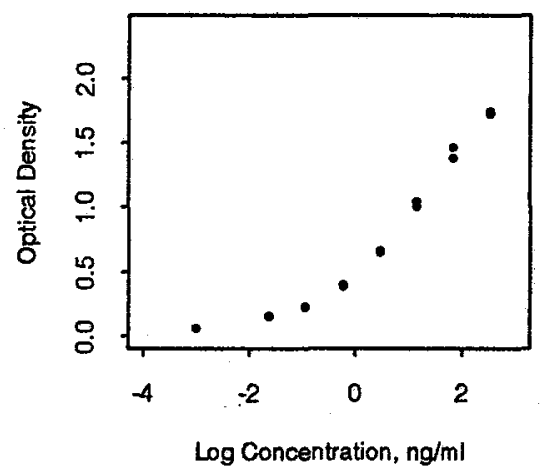

Assay 11

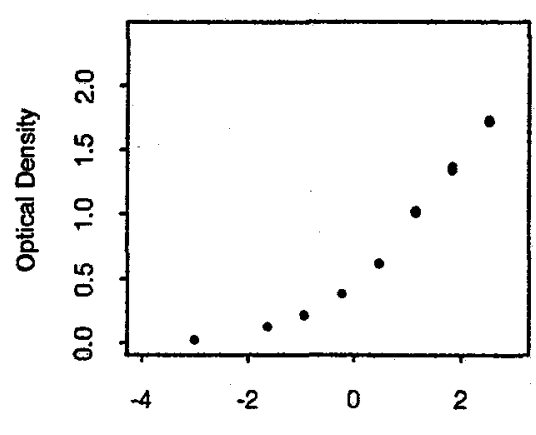

$\log$ Concentration, ng/ml

Assay 3

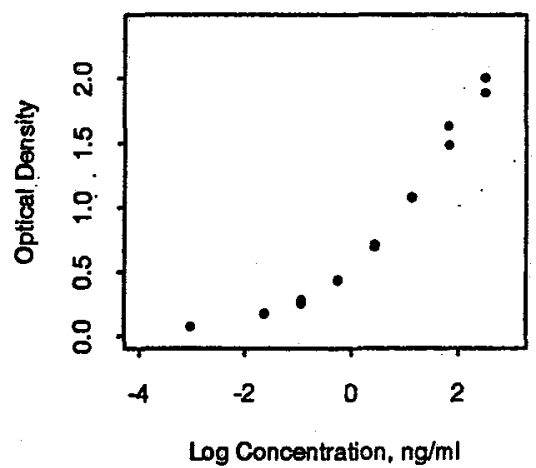

Assay 6

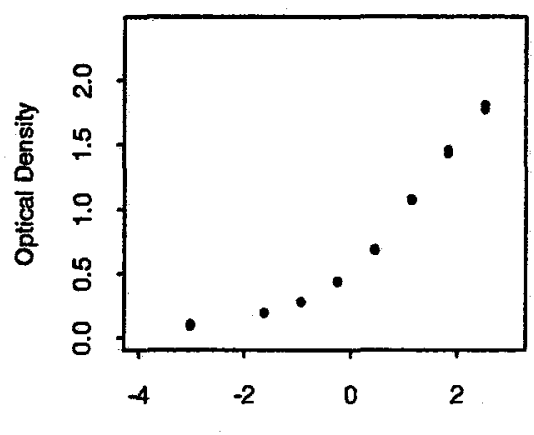

Log Concentration, ng/mi

Assay 9

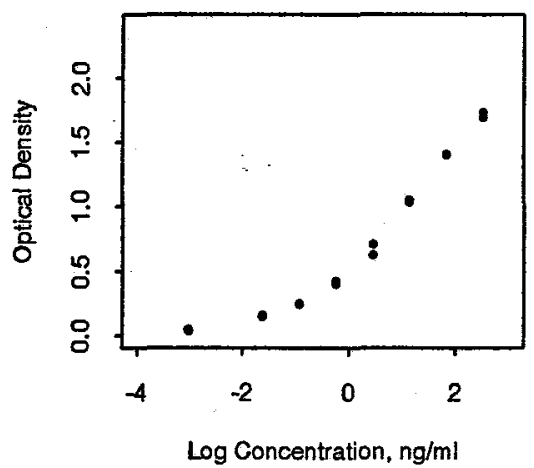


Figure 2 : Relaxin NHE Cell Bioassay

Assay 1

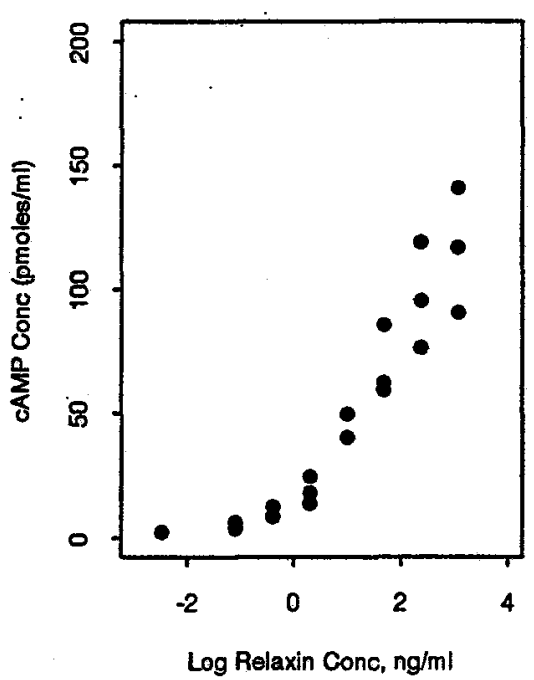

Assay 4

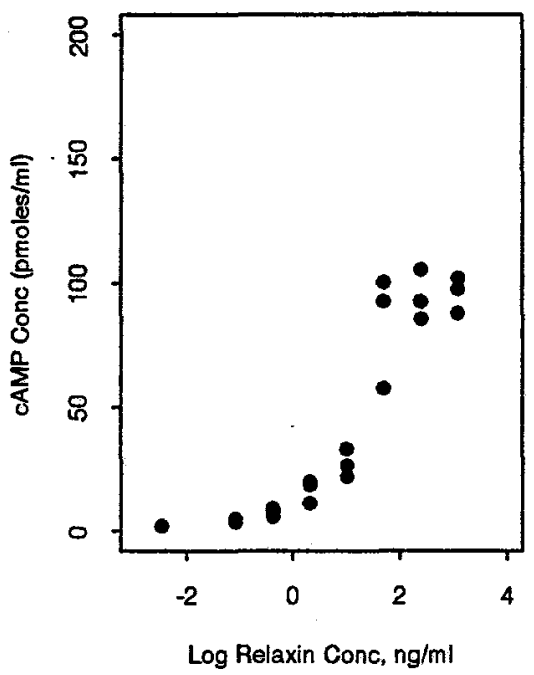

Assay 7

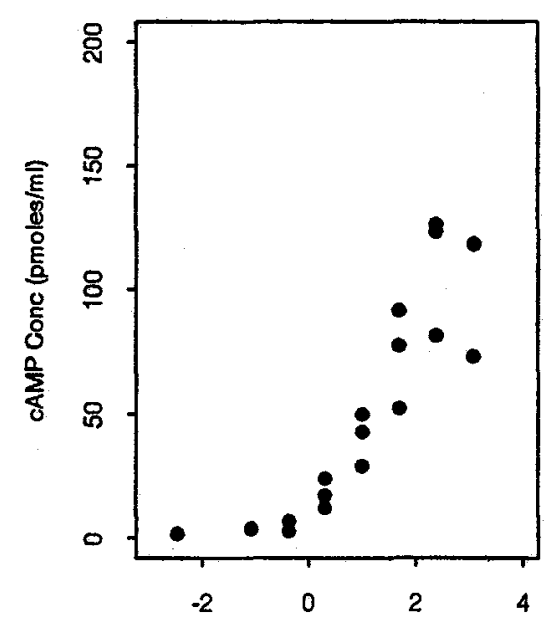

Log Relaxin Conc, ng/ml
Assay 2

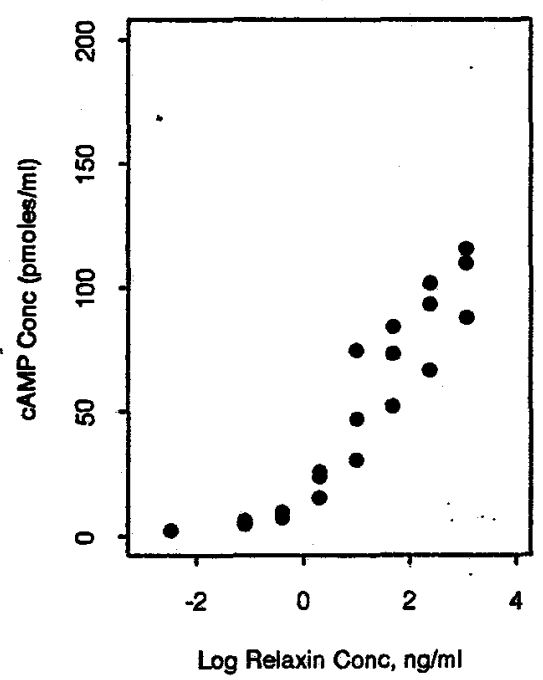

Assay 5

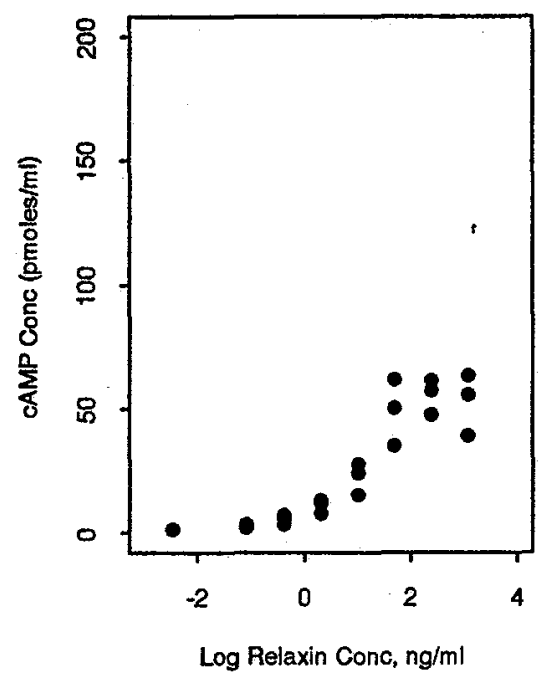

Assay 8

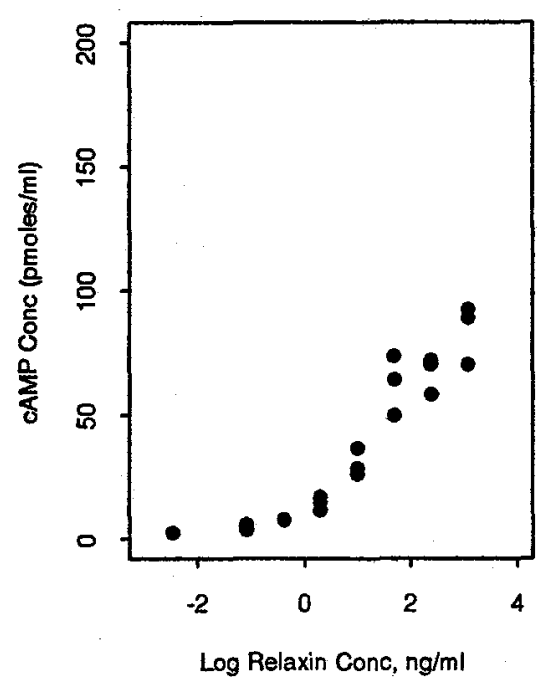

Assay 3

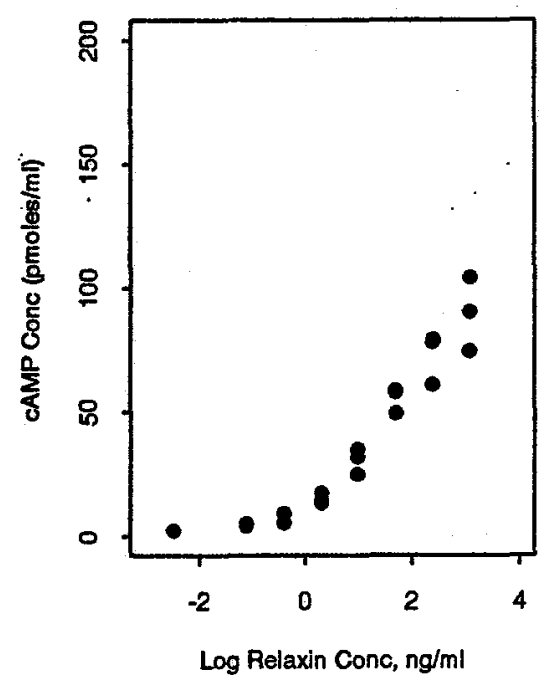

Assay 6

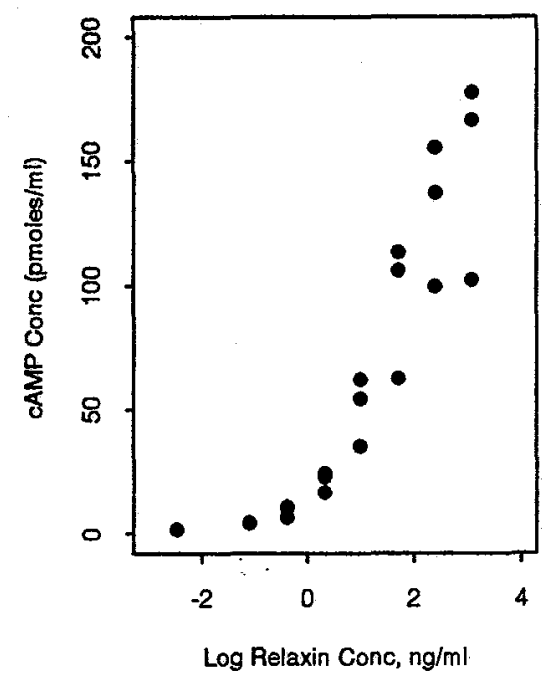

Assay 9

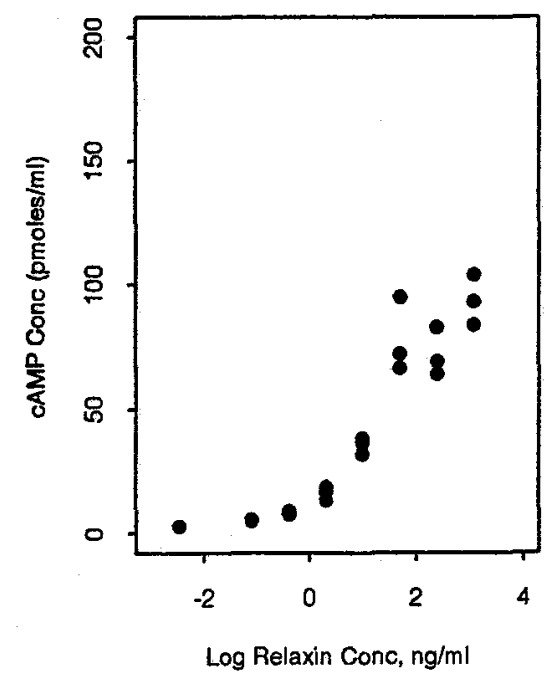


Figure 3

Relaxin Assay 1 : Precision Profiles for Different Theta Values

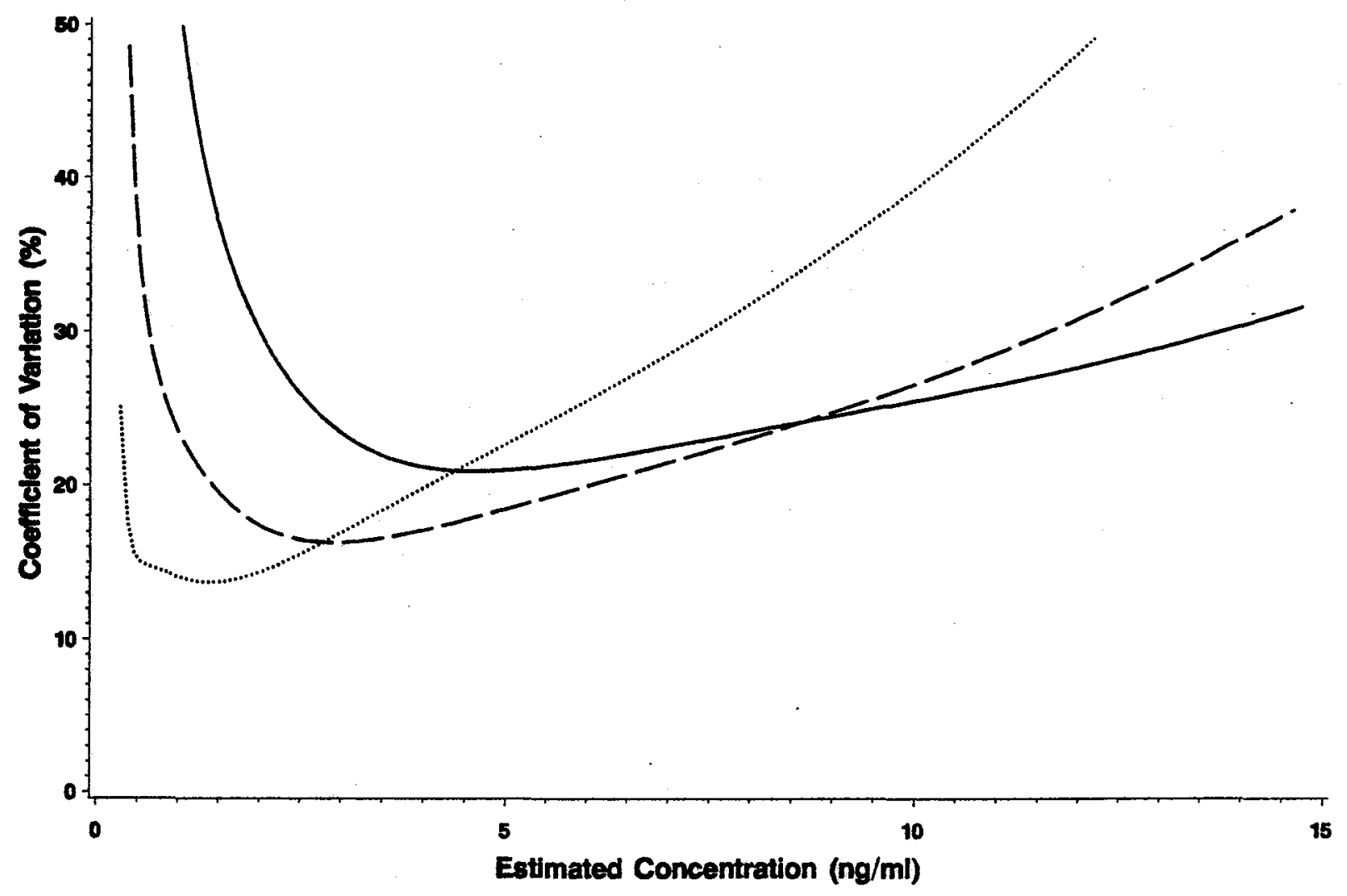

THETA $-0.0-0.8 \quad$............. 1.0

Figure 4

Relaxin Assay 1 : Precision Profiles Under Different Assumptions

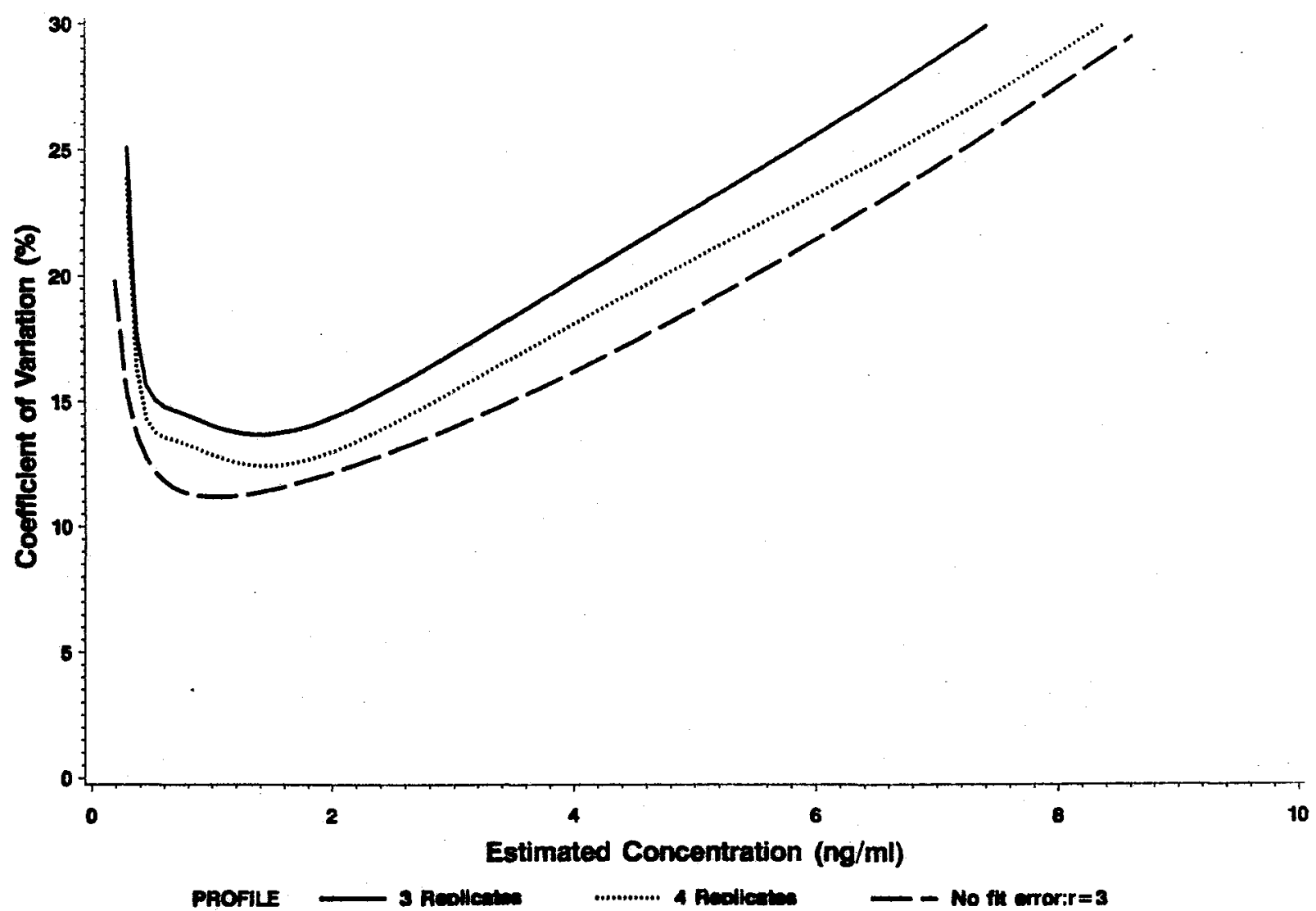


Figure 5a : Relaxin Bioassay

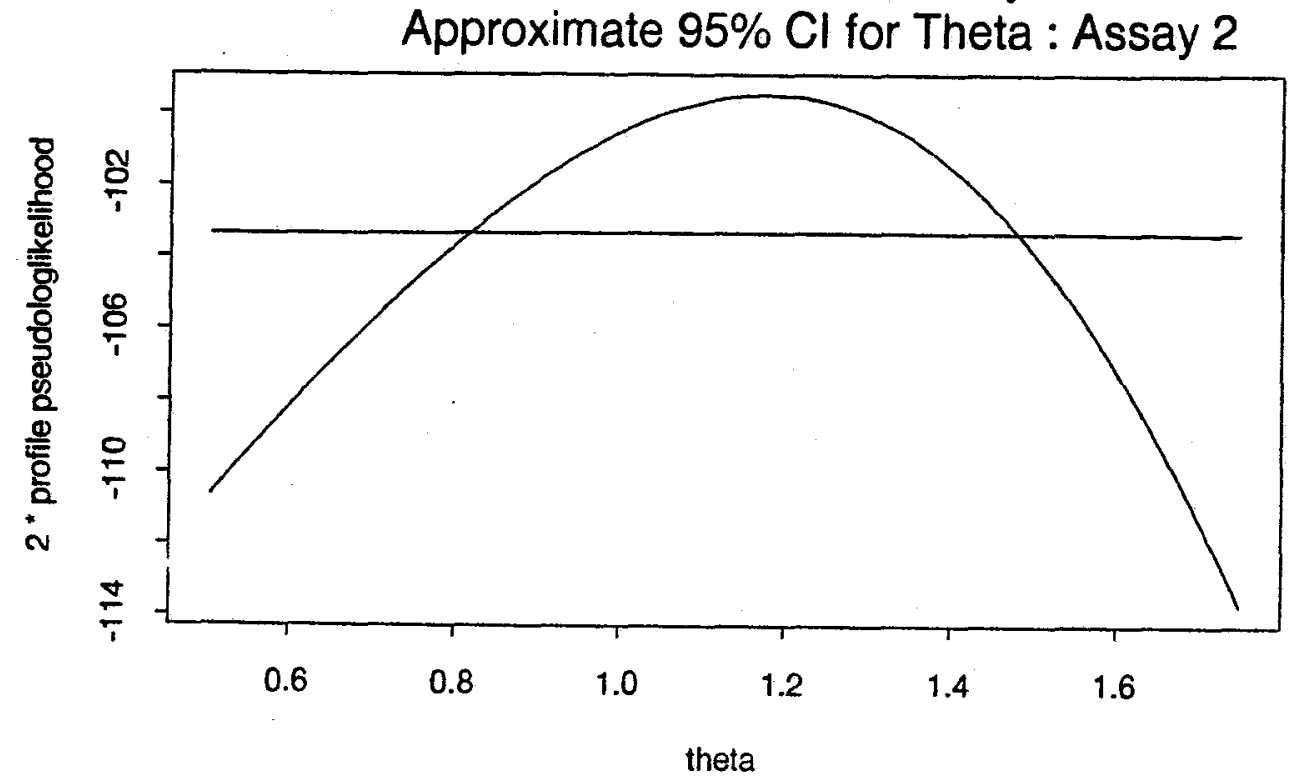

Figure 5b : Relaxin Bioassay

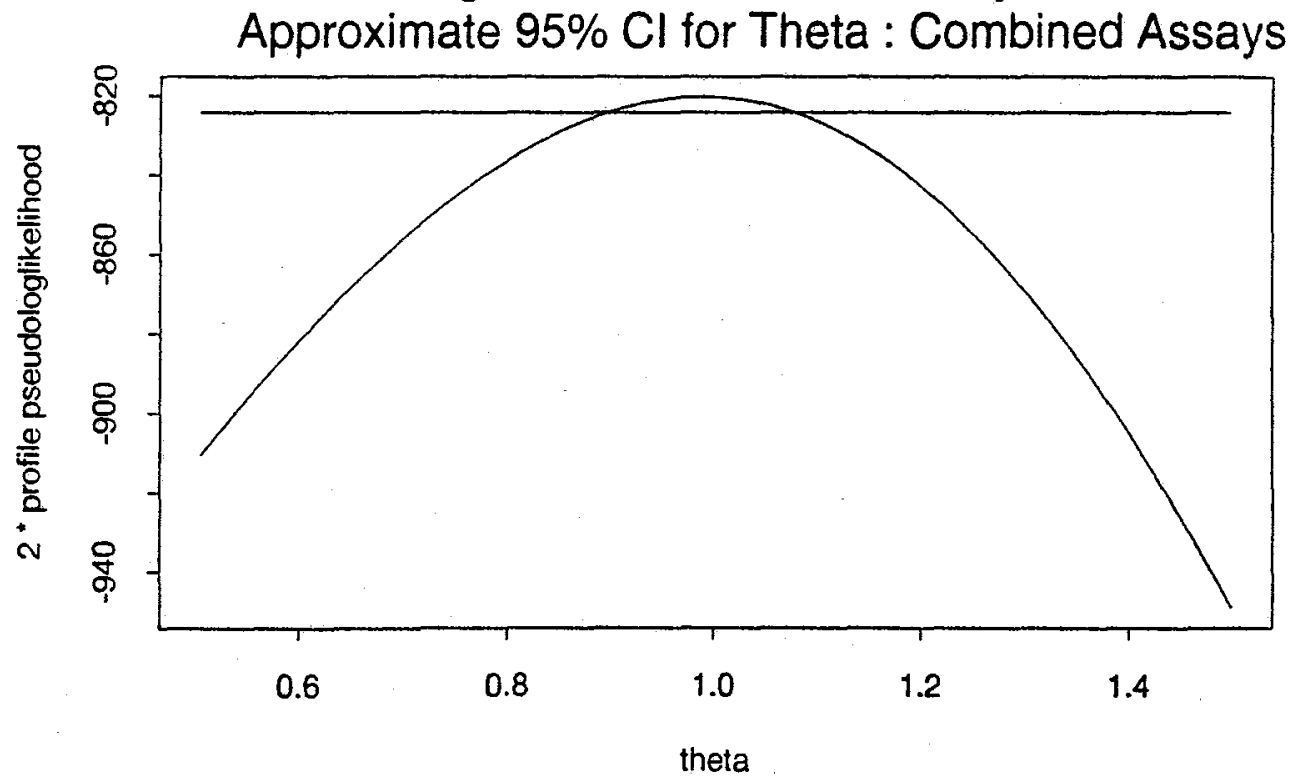


Figure 6a : DNase ELISA 95\% Confidence Intervals Assay 2

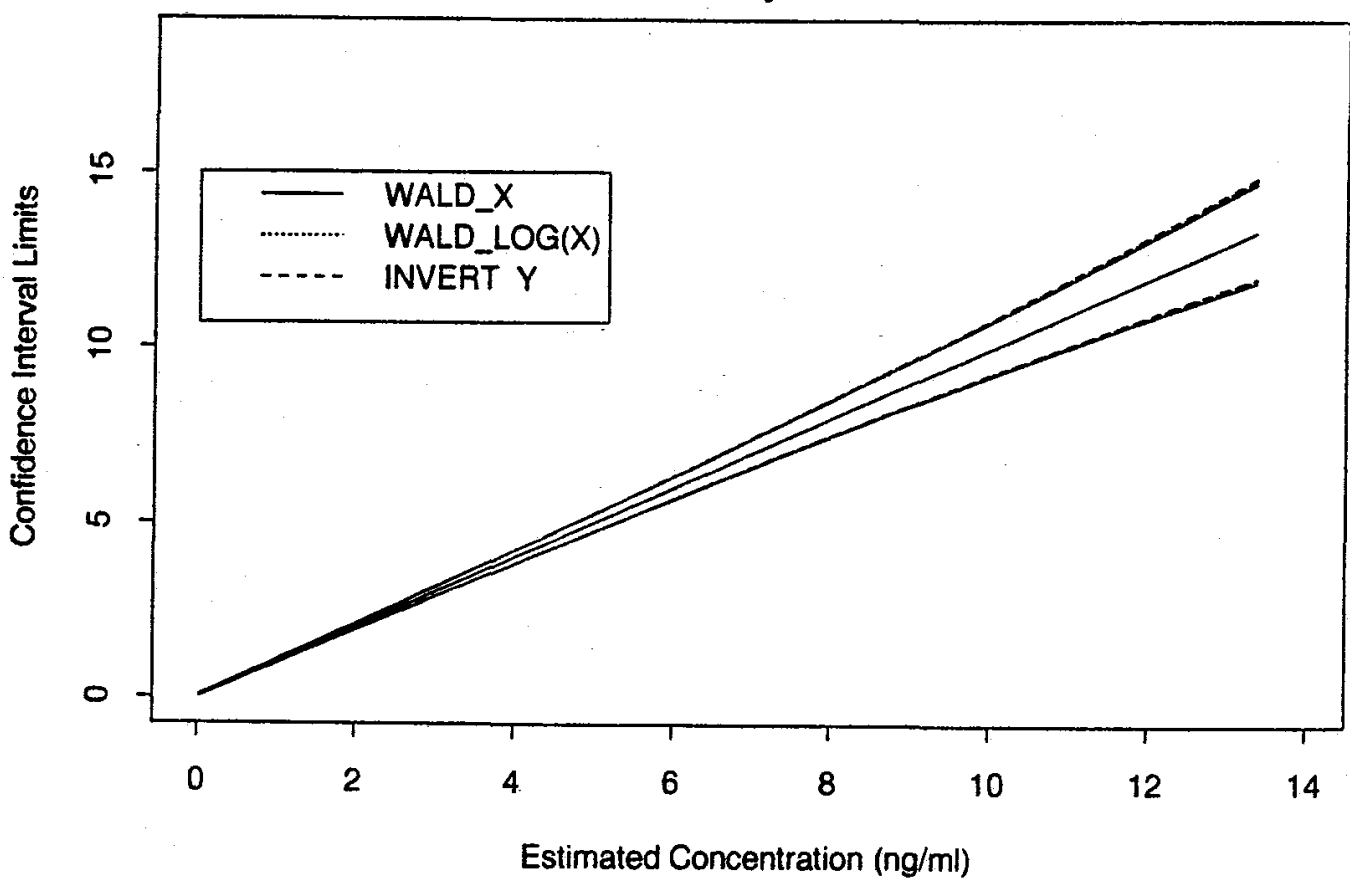

Figure $6 b$ : Relaxin Bioassay 2 : 95\% Confidence Intervals

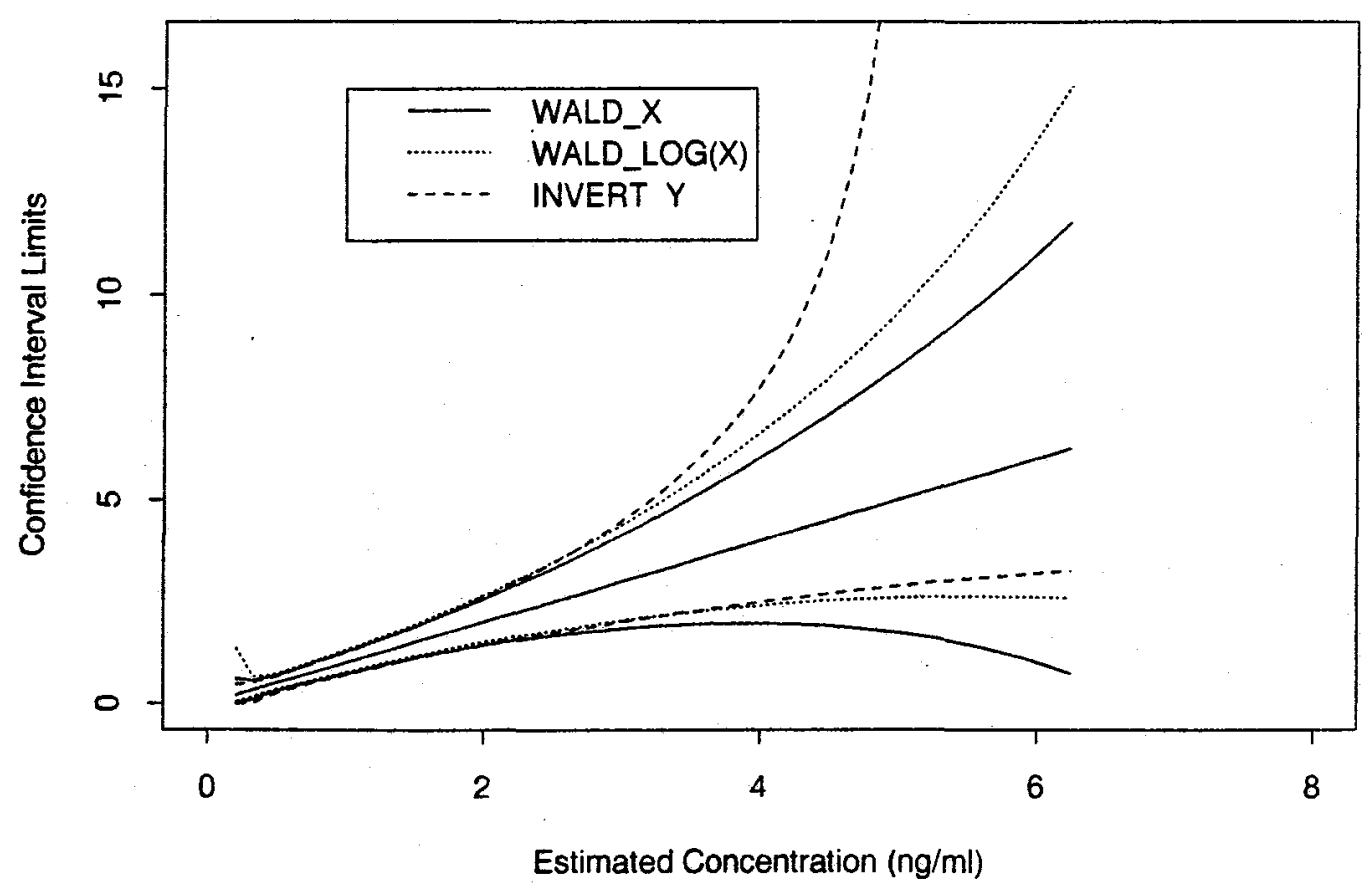

